ARTICLE

\title{
Structural basis of neuropeptide $Y$ signaling through Y1 receptor
}

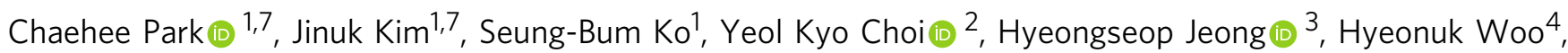
Hyunook Kang (1) ${ }^{1}$, Injin Bang (10 1,6, Sang Ah Kim ${ }^{1,5}$, Tae-Young Yoon (1) ${ }^{1,5}$, Chaok Seok (i) ${ }^{4}$, Wonpil Im (1) ${ }^{2} \&$ Hee-Jung Choi (iD ${ }^{1 凶}$

Neuropeptide Y (NPY) is highly abundant in the brain and involved in various physiological processes related to food intake and anxiety, as well as human diseases such as obesity and cancer. However, the molecular details of the interactions between NPY and its receptors are poorly understood. Here, we report a cryo-electron microscopy structure of the NPY-bound neuropeptide $Y 1$ receptor $\left(Y_{1} R\right)$ in complex with $G_{i 1}$ protein. The NPY $C$-terminal segment forming the extended conformation binds deep into the $Y_{1} R$ transmembrane core, where the amidated C-terminal residue $Y 36$ of NPY is located at the base of the ligand-binding pocket. Furthermore, the helical region and two $\mathrm{N}$-terminal residues of NPY interact with $Y_{1} R$ extracellular loops, contributing to the high affinity of NPY for $Y_{1} R$. The structural analysis of NPY-bound $Y_{1} R$ and mutagenesis studies provide molecular insights into the activation mechanism of $Y_{1} R$ upon NPY binding.

\footnotetext{
${ }^{1}$ Department of Biological Sciences, Seoul National University, Seoul 08826, Republic of Korea. ${ }^{2}$ Departments of Biological Sciences and Chemistry, Lehigh University, Bethlehem, PA 18015, USA. ${ }^{3}$ Center for Electron Microscopy Research, Korea Basic Science Institute, Chungcheongbuk-do 28119, Republic of Korea. ${ }^{4}$ Department of Chemistry, Seoul National University, Seoul 08826, Republic of Korea. ${ }^{5}$ Institute for Molecular Biology and Genetics, Seoul National University, Seoul 08826, Republic of Korea. ${ }^{6}$ Present address: Perlmutter Cancer Center, New York University Langone Health, and Department of Biochemistry and Molecular Pharmacology, New York University School of Medicine, New York 10016 NY, USA. ${ }^{7}$ These authors contributed equally: Chaehee Park, Jinuk Kim. ${ }^{凶}$ email: choihj@snu.ac.kr
} 


\section{T} he human neuropeptide Y (NPY) system comprises three peptide ligands, NPY, peptide YY (PYY), and pancreatic polypeptide (PP), and four functional NPY receptors $Y_{1} R$, $\mathrm{Y}_{2} \mathrm{R}, \mathrm{Y}_{4} \mathrm{R}$, and $\mathrm{Y}_{5} \mathrm{R}^{1}$. These endogenous peptide ligands consisting of 36 amino acids with the amidated $\mathrm{C}$-terminus activate specific NPY receptors generally coupled to $G_{i}$ or $G_{o}$ protein ${ }^{2}$. Of these three peptide ligands, NPY, a highly abundant peptide ligand in the brain, can activate all four subtypes of the NPY receptor and is involved in various physiological processes such as food intake, stress response, anxiety, and memory retention ${ }^{3-5}$. Furthermore, NPY signaling is involved in human diseases such as obesity, mood disorders, and cancers ${ }^{6-8}$.

The nuclear magnetic resonance (NMR) structure of NPY reveals that its C-terminal segment (13-36) forms an amphipathic $\alpha$-helix, and the remaining $\mathrm{N}$-terminal part is unstructured and flexible ${ }^{9,10}$. Previous functional assays using the $\mathrm{N}$-terminal truncation mutants of NPY indicate that the complete $\mathrm{N}$-terminus of NPY is necessary for $\mathrm{G}_{\mathrm{i}}$ signaling through $\mathrm{Y}_{1} \mathrm{R}$ but not through $\mathrm{Y}_{2} \mathrm{R}$, which shares approximately $30 \%$ sequence identity with $\mathrm{Y}_{1} \mathrm{R}^{10,11}$. Similarly, PYY, highly homologous to NPY, binds to $Y_{1} R$ and $Y_{2} R$ in its full-length form; however, PYY (3-36), the N-terminal cleaved form found in the circulation, binds only to $\mathrm{Y}_{2} \mathrm{R}^{12-14}$.

NPY receptors belong to the $\beta$ subgroup of class A G proteincoupled receptors (GPCRs). Among them, $\mathrm{Y}_{1} \mathrm{R}$ is expressed in the central nervous system (CNS) as well as in the adipose tissue and vascular smooth muscle cells, where it leads to enhanced cell proliferation and the induction of food intake upon activation by NPY ${ }^{15-17}$. Thus, the $Y_{1} R$ antagonist has been proposed as a potential drug for treating obesity ${ }^{16}$. Furthermore, $Y_{1} R$ is highly expressed in human primary breast cancer, implying the utility of $\mathrm{Y}_{1} \mathrm{R}$ as a diagnostic marker for breast cancer ${ }^{18}$.

The crystal structures of the small molecule antagonist-bound $\mathrm{Y}_{1} \mathrm{R}$ have been published recently ${ }^{10}$. Although these structures provide molecular details of $\mathrm{Y}_{1} \mathrm{R}$-selective antagonist binding and the overall architecture of an inactive state of $Y_{1} R$, the molecular mechanism of its activation by binding of the endogenous agonist NPY is still unknown.

Here, we present a single-particle cryo-electron microscopy (cryo-EM) structure of NPY-bound wild-type $\mathrm{Y}_{1} \mathrm{R}$ with $\mathrm{G}_{\mathrm{i} 1}$ protein coupled. The structure reveals that five amino acids at the C-terminus of NPY form an extended conformation and are inserted into a pocket formed by the transmembrane (TM) domain of $Y_{1} R$. In addition, the helical region and $\mathrm{N}$-terminus of NPY are shown to be involved in $Y_{1} R$ binding. Together with molecular dynamics (MD) simulations and functional analysis of various mutations, our structure provides molecular details of endogenous peptide recognition by $\mathrm{Y}_{1} \mathrm{R}$ and suggests the activation mechanism of $\mathrm{Y}_{1} \mathrm{R}$ upon NPY binding.

\section{Results}

Structure determination of the NPY $-Y_{1} R-G_{i 1}$ complex. The published antagonist-bound crystal structure of $\mathrm{Y}_{1} \mathrm{R}$ was solved using a modified construct involving thermostabilizing mutation, C-terminal truncation, and replacement of intracellular loop 3 (ICL3) with T4 lysozyme ${ }^{10}$. In our study, we used the wild-type $\mathrm{Y}_{1} \mathrm{R}$ construct (2-384) with minimal engineering (such as affinity tag) to solve the receptor structure, thereby facilitating molecular analysis of the activation mechanism of the native receptor. $Y_{1} R$ is known to couple $G_{i 1}$ protein to activate downstream signaling upon NPY binding ${ }^{2}$. Before purifying the NPY- ${ }_{1} R-G_{i 1}$ complex, we confirmed the functionality of the synthesized NPY by performing bioluminescence resonance energy transfer (BRET) assays, which showed that $Y_{1} R$ specifically recruited $G_{i 1}$ in response to NPY binding (Supplementary Fig. 1). For the structural study, $Y_{1} R$ and $G_{i 1}$ heterotrimer were purified separately and mixed in the presence of NPY. The complex was incubated with apyrase to obtain a nucleotide-free $\mathrm{G}_{\mathrm{i} 1}$ heterotrimer, and single-chain variable fragment termed scFv16, that specifically recognizes heterotrimeric $G_{i}$ was added as a stabilizer ${ }^{19}$ (Supplementary Fig. 2).

We determined a cryo-EM structure of the NPY-Y ${ }_{1} R-G_{i 1}-s c F v 16$ complex at a nominal resolution of $3.2 \AA$ in glyco-diosgenin (GDN) micelles (Supplementary Table 1 and Supplementary Fig. 3). Similar to other structures of nucleotide-free $G$ protein heterotrimers bound to GPCR, the $\alpha$-helical domain of $\mathrm{G}_{\mathrm{i} 1}$ was not modeled in our structure because of its flexibility. Inspection of the cryo-EM map clearly showed the density of NPY protruding into the extracellular region (Fig. 1a). To further focus on the binding of NPY to $Y_{1} R$, we subtracted the heterotrimeric $G$ protein signal and subjected to local refinement (Supplementary Fig. 3) as used in the cryo-EM analysis of secretin-bound secretin receptor $-\mathrm{G}_{\mathrm{s}}$ complex ${ }^{20}$. The resulting cryo-EM map allowed modeling of the five residues at the N-terminus (1-5) and the C-terminal half of NPY (20-36) (Fig. 1b, $\mathrm{c}$ and Supplementary Fig. 4).

Overall structure of NPY-bound $Y_{1} R$ receptor. Structural comparison of the NPY-bound and antagonist-bound $\mathrm{Y}_{1} \mathrm{R}$ reveals distinct conformational changes in the extracellular region, TM core, and cytoplasmic part of the receptor upon activation. As the amidated C-terminus of NPY penetrates deep into the TM core, the extracellular tips of TM3, TM4, TM6, and TM7 slightly move outward by 1.9-2.5 $\AA$ in the NPY-bound $\mathrm{Y}_{1} \mathrm{R}$ structure compared to the antagonist-bound structure, opening up the ligand-binding pocket (Fig. 2a). In fact, the calculated solvent-accessible ligandbinding cavities in the antagonist-bound and NPY-bound structures are $\sim 506$ and $730 \AA^{3}$, respectively (Supplementary Fig. 5). Besides the interaction of the C-terminal tail of NPY with the $Y_{1} R$ TM core, the molecular interaction of the remaining NPY with $\mathrm{Y}_{1} \mathrm{R}$ was difficult to characterize, as the NPY map was not well resolved (Supplementary Fig. 3). However, we could observe the density of the N-terminal and helical regions of NPY, which are surrounded by extracellular loops (ECLs) and the N-terminal region of $\mathrm{Y}_{1} \mathrm{R}$ (Supplementary Fig. 6). ECL2, ECL3, and the $\mathrm{N}$-terminal region of $\mathrm{Y}_{1} \mathrm{R}$ directly interact with NPY to varying degrees. The details of the NPY interaction are discussed later.

NPY binding results in the TM core of $\mathrm{Y}_{1} \mathrm{R}$ undergoing conventional conformational changes associated with the activation mechanism of class A GPCRs. Immediately below the C-terminus of NPY peptide, side chains of $\mathrm{I} 128^{3.40}, \mathrm{P} 223^{5.50}$, and F272 6.44 (superscripts are the Ballesteros-Weinstein numbers ${ }^{21}$ ) in the connector region are repacked to contract the interface of TM3, 5, and 6 (Fig. 2b), resulting in the outward movement of TM6 and inward movement of TM7 at the cytoplasmic part of $\mathrm{Y}_{1} \mathrm{R}$ (Fig. 2a). In addition, $\mathrm{R} 138^{3.50}$ of the $(\mathrm{D} / \mathrm{E}) \mathrm{R}(\mathrm{Y} / \mathrm{H})$ motif makes close contact with $\mathrm{Y} 231^{5.58}$ and $\mathrm{Y} 320^{7.53}$ of the NPxxY motif (Fig. 2c), a well-known key interaction observed in the activated GPCR structures. These conformational changes demonstrate that our structure represents the active conformation of class A GPCRs.

Compared to other $\mathrm{G}_{\mathrm{i} 1}$-bound class A GPCRs, such as neurotensin receptor 1 (NTSR1) and $\mu$-opioid receptor ( $\mu \mathrm{OR})$, $\mathrm{Y}_{1} \mathrm{R}$ has a relatively short ICL2, comprising six amino acids, including two Pro residues (Fig. 2d). In NTSR1 and $\mu$ OR, ICL2s adopt an $\alpha$-helix upon activation, interacting with the $\alpha \mathrm{N}, \alpha \mathrm{N}-\beta 1$ loop and $\alpha 5$ helix of $G_{i 1} 22,23$. In contrast, ICL2 of $Y_{1} R$ remains as a loop in the $G_{i 1}$-bound state, forming contacts with only the $\alpha 5$ helix of $G_{i}$ through R146 ${ }^{\text {ICL2 }}$ and R149 ICL2 (Fig. 2d). In addition to ICL2, hydrophobic residues in TM3, TM5, and TM6 (I142 3.54 $\mathrm{I} 234^{5.61}, \mathrm{~L} 238^{5.65}, \mathrm{I} 261^{6.33}$, and $\mathrm{L} 265^{6.37}$ ) of $\mathrm{Y}_{1} \mathrm{R}$ form van der 
a
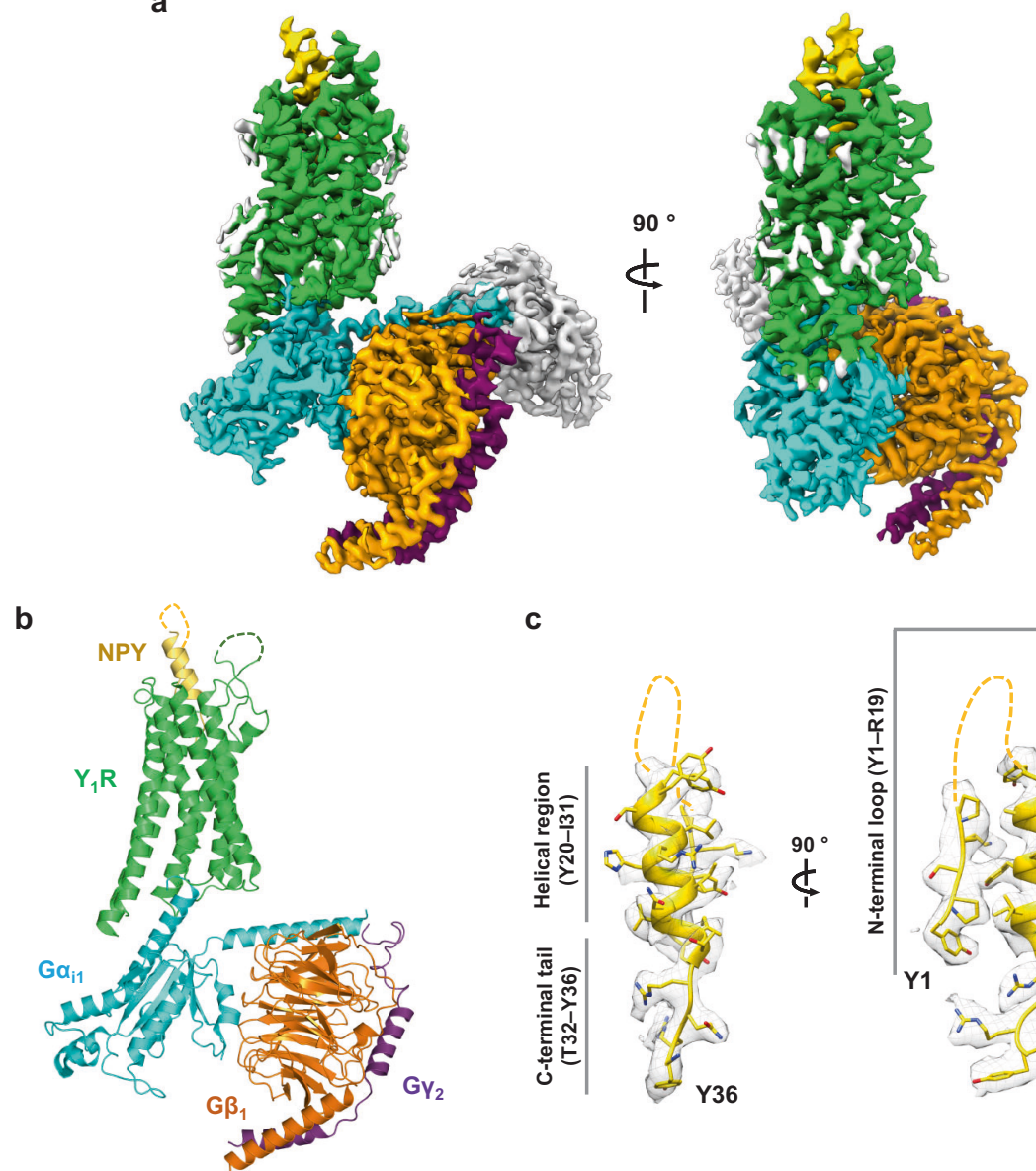

C
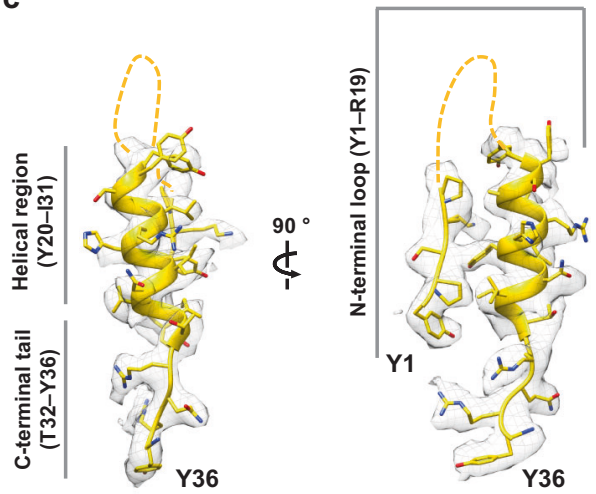

Fig. 1 Overall structure of the $\mathbf{N P Y}-\mathbf{Y}_{\mathbf{1}} \mathbf{R}-\mathbf{G}_{\mathbf{i 1}}-\mathbf{s c F v 1 6}$ complex. a The cryo-EM map of the NPY-Y $\mathrm{R}_{1}-\mathrm{G}_{i 1}-\mathrm{scFv} 16$ complex is shown. $Y_{1} R, N P Y, G \alpha_{i 1}, G \beta_{1}, G \gamma_{2}$, and scFv16 are colored green, yellow, cyan, orange, purple, and gray, respectively. The remaining micelle density is shown in light gray. Details on cryo-EM map generation are described in the "Methods" section. $\mathbf{b}$ The structure of the NPY-Y $R-G_{i 1}$ complex is shown. scFv16 is included in the final structure but omitted in this figure for clarity. c The sharpened cryo-EM map with the NPY peptide model is shown in two orientations. NPY residues 6-19 are not modeled in this structure and are shown as dashed lines. The three regions of NPY are marked as the N-terminal loop region (Y1-R19), the helical region (Y20-131), and the C-terminal tail (T32-Y36).

Waals interactions with L348 and L353 in the a5 helix of $\mathrm{G}_{\mathrm{i} 1}$ (Supplementary Fig. 7) and $\mathrm{R} 260^{6.32}$ of $\mathrm{Y}_{1} \mathrm{R}$ forms polar interaction with the carboxyl group of F354 in the a5 helix of $\mathrm{G}_{\mathrm{i} 1}$ (Supplementary Fig. 7). Most of these interactions are conserved in the hNTSR1-G $\mathrm{G}_{\mathrm{i} 1}$ and $\mu \mathrm{OR}-\mathrm{G}_{\mathrm{i} 1}$ structures (Supplementary Fig. 7). However, when aligning the receptors, the relative position of the $a 5$ helix of $G_{i 1}$ bound to $Y_{1} R$ slightly differs by $\sim 2 \AA$ displacement of the C-terminus of $\mathrm{Ga}_{\mathrm{i} 1}$ or $\sim 8^{\circ}$ tilt angle of the $a 5$ helix of $G_{i 1}$ in the NTSR1-bound and $\mu$ OR-bound $\mathrm{G}_{\mathrm{i} 1}$ structures, respectively (Supplementary Fig. 7).

Binding of the C-terminal tail of NPY to $Y_{1} R$. Our cryo-EM map shows a well-resolved density for the five C-terminal residues of NPY (32-36) (Fig. 1c), forming an extended structure, contrasting the $a$-helix formation of residues $13-36$ in the NMR structure of human NPY (PDB ID 1 RON $)^{9}$. However, helix unwinding at the C-terminal tail of NPY was not unexpected since the previous NMR study of porcine NPY bound to $\mathrm{Y}_{1} \mathrm{R}$ suggested an extended conformation of the NPY C-tail ${ }^{10}$. Similarly, the nine amino acids at the $\mathrm{C}$-terminus of orexin-B neuropeptide $(\mathrm{OxB})$ were previously shown to form an extended conformation in the orexin receptor $\left(\mathrm{OX}_{2} \mathrm{R}\right)$-bound state but form an $\alpha$-helix in the receptor-free state ${ }^{24,25}$.
The extended conformation of the NPY C-tail binds to a pocket lined by TM helices $2-7$, with a depth of $\sim 11 \AA$ from the top surface of the membrane (Fig. 3a, b). It is well established that the amidation of NPY C-terminal tyrosine is critical for its function; consistently, in this study, non-amidated NPY failed to elicit G protein signaling (Supplementary Fig. 8). At the bottom of the ligand-binding pocket, the C-terminal amide of NPY points toward the side chain of Q1203.32 (Fig. 3c), which has been predicted to interact with the Y36 side chain in the NPY-docked $\mathrm{Y}_{1} \mathrm{R}$ model ${ }^{10}$. The importance of Q1203.32 for $\mathrm{G}_{\mathrm{i}}$ recruitment and signaling upon NPY treatment was investigated using BRET and calcium signaling assays. As expected, the Q120 $0^{3.32} \mathrm{~A}$ mutant exhibited reduced $\mathrm{Ga}_{\mathrm{i} 1}$ recruitment and an increased $\mathrm{EC}_{50}$ (Supplementary Table 2 and Supplementary Figs. 9, 10, and 11). The C-terminal amide of Y36 is further coordinated by $\mathrm{H} 306^{7.39}$ through polar interactions and $\mathrm{C} 93^{2.57}$ and M310 7.43 through van der Waals contacts (Fig. 3c). Furthermore, the ligand-binding pocket of $\mathrm{Y}_{1} \mathrm{R}$ is highly acidic (Supplementary Fig. 12). The acidic residues of the binding pocket repel the negatively charged C-terminus and favor the amidated C-terminus. A similar acidic patch in the ligand-binding pocket is observed in $\mathrm{OX}_{2} \mathrm{R}$, where the amidated $\mathrm{C}$-terminus of $\mathrm{OxB}$ binds. In contrast, the ligand-binding pocket is basic in NTSR1 and endothelin $\mathrm{B}$ receptor $\left(\mathrm{ET}_{\mathrm{B}} \mathrm{R}\right)$, whose peptide agonists have the C-terminal carboxyl groups (Supplementary Fig. 12). 
a
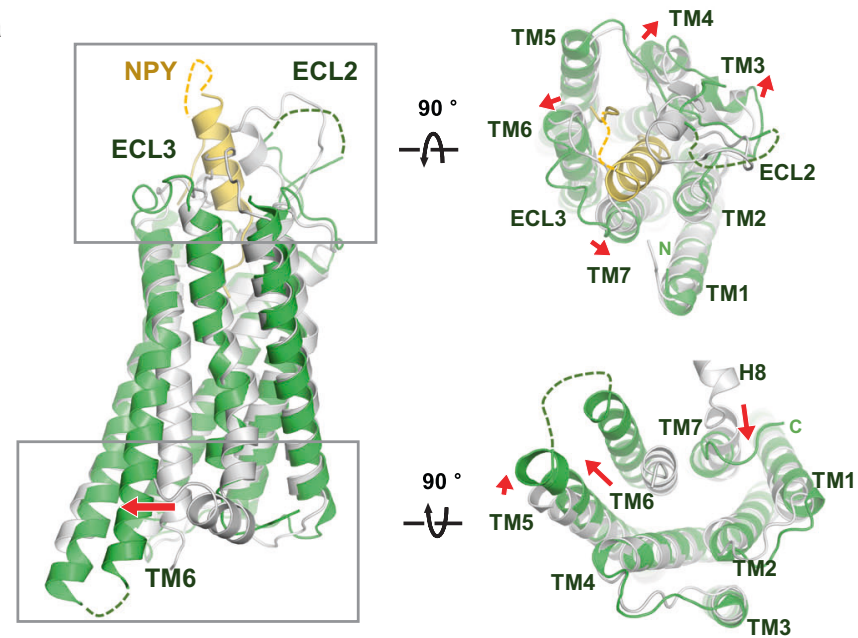

b

C

d
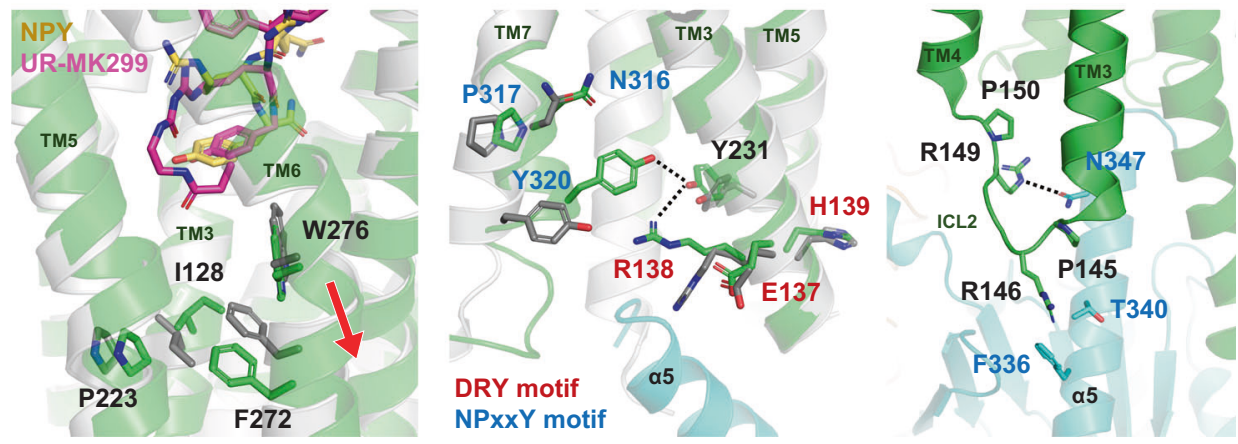

Fig. 2 Comparison between NPY-bound and antagonist-bound $\mathbf{Y}_{\mathbf{1}} \mathbf{R}$ structures. a Structural alignment between NPY (yellow)-bound $Y_{1} R$ (current structure, green) and antagonist-bound $Y_{1} R$ (PDB ID 5ZBQ, light gray, residues 18-32 of $Y_{1} R$ and antagonist are omitted for clarity) clearly shows characteristic TM6 movement as observed in the active structures of class A GPCRs. Views from extracellular (right-upper panel) and cytoplasmic (rightlower panel) sides are shown on the right. Each red arrow represents the movement of the TM helix. $\mathbf{b}$ Structural changes at the connector region (P5.50/3.40F6.44 motif) upon NPY binding are shown. The antagonist-bound $Y_{1} R$ (PDB ID 5ZBQ) is colored in light gray and an antagonist, UR-MK299, in magenta. c Structural changes at the $D(E) / R / Y(H)$ (labeled in red) and NPxxY (labeled in blue) motifs are shown. $\mathbf{d}$ The binding interface between $\alpha 5$ of $\mathrm{G}_{\mathrm{i} 1}$ (in cyan) and ICL2 is shown. Residues participating in the interactions and two Pro residues (P145 ICL2 and P150ICL2) in ICL2 are shown as sticks. Dashed lines represent the polar interactions.

The Y36 side chain of NPY forms a hydrogen bond with Q2195.46 through its hydroxyl group and hydrophobic interaction with $1124^{3.36}$ through its phenyl ring (Fig. 3c). The importance of Q2195.46 and I124.36 for NPY signaling was demonstrated by 13.5-fold and 3.5-fold reduction in NPY potency in the Q2195.46 A and $\mathrm{I} 124^{3.36} \mathrm{~A}$ mutants, respectively ${ }^{10}$. Previous mutational studies of NPY showed that the Y36F mutation had a relatively mild effect on $\mathrm{Y}_{1} \mathrm{R}$ binding ${ }^{26}$. In contrast, the $\mathrm{Y} 36 \mathrm{~A}$ mutation caused a loss of binding to $Y_{1} R^{10,26}$, suggesting that the phenyl ring of Y36 is more important for NPY signaling. Our structure shows that the phenyl ring of Y36 forms an intramolecular interaction with R35 of NPY (Supplementary Fig. 13), which aids in correctly positioning R35, in addition to hydrophobic interactions with $\mathrm{Y}_{1} \mathrm{R}$ (Fig. 3a).

R33 and R35 of NPY extensively interact with the $Y_{1} R$ TM core, and alanine scanning mutagenesis of NPY has shown that R33A and R35A mutations of NPY exhibit the most severe defects in $\mathrm{Y}_{1} \mathrm{R}$ binding ${ }^{10,26}$. R33 of NPY forms a hydrogen bond with $\mathrm{N} 283^{6.55}$ and $\pi$-cation interactions with $\mathrm{F} 286^{6.58}$ and F3027.35 (Fig. 3d). BRET analysis using the N2836.55 A mutant showed a dramatic decrease in $\mathrm{Ga}_{i}$ recruitment and reduced NPY potency by 85 -fold compared to wild-type $\mathrm{Y}_{1} \mathrm{R}$ (Supplementary Table 2 and Supplementary Figs. 9, 10, and 11). Previous mutagenesis studies of $\mathrm{F} 286^{6.58}$ and $\mathrm{F} 302^{7.35}$ showed that substituting of these residues with alanine caused a reduction in
NPY potency ${ }^{10,27}$. R35 forms electrostatic interaction with $\mathrm{D} 287^{6.59}$ and van der Waals interaction with $\mathrm{F} 173^{4.60}$, both of which have been reported to be essential for NPY signaling ${ }^{10,28}$.

Q34 and T32 of NPY are oriented opposite to the R35 and R33 side chains, interacting with $\mathrm{T} 97^{2.61}, \mathrm{Y} 100^{2.64}$, and the backbone carbonyl of D104 2.68 (Fig. 3e). In particular, Y100 2.64 is sandwiched between T32 and Q34, forming nonpolar contacts with both (Fig. 3e). Consistent with these structural data, $\mathrm{Y} 100^{2.64} \mathrm{~A}$, a well-known mutation, dramatically decreased downstream NPY signaling ${ }^{10,27,28}$. In the antagonist-bound structures, none of these residues that interact with T32 and Q34 are involved in antagonist binding.

Altogether, our structural analysis shows that the C-terminal tail of NPY forms extensive interactions with $\mathrm{Y}_{1} \mathrm{R}$ residues on TM2, 3, 5, and 6. Previous and current mutational studies support the importance of these interacting residues in NPY signaling.

Structural changes in $Y_{1} R$ TM core by binding of NPY C-terminal tail. The binding pocket for the NPY C-terminus is where the two antagonists (UR-MK299 and BMS-193885) bind in the previously reported inactive $\mathrm{Y}_{1} \mathrm{R}$ structures. The structural comparison reveals that the hydroxyphenyl group and guanidine moiety of UR-MK299 exhibit binding modes similar to those of the side chains of Y36 and R35 of NPY, respectively, making polar interactions with Q2195.46 and D2876.59 (Fig. 4a). The BMS- 
a

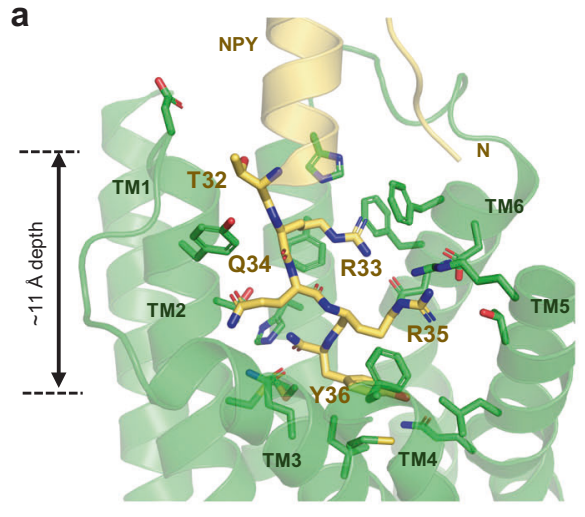

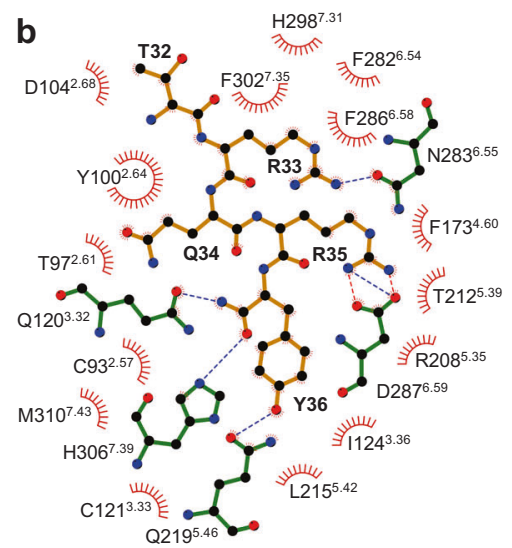

C

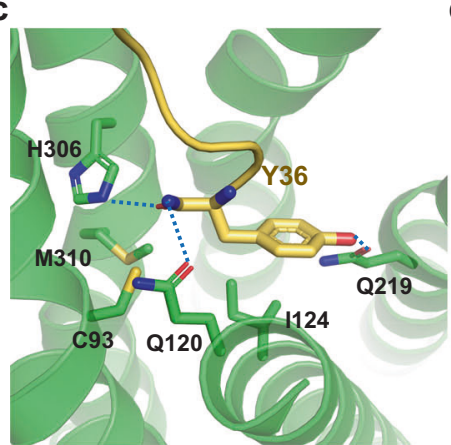

d

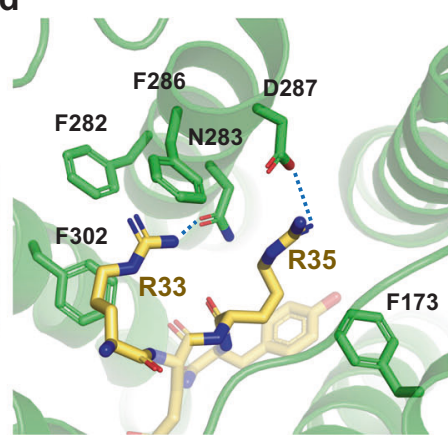

e

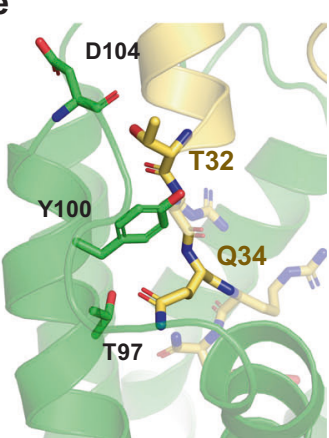

Fig. 3 Binding of NPY C-terminal tail to $\mathbf{Y}_{\mathbf{1}} \mathbf{R}$. a The C-terminal tail of NPY (yellow) and $Y_{1} R$ residues that participate in NPY binding are presented as

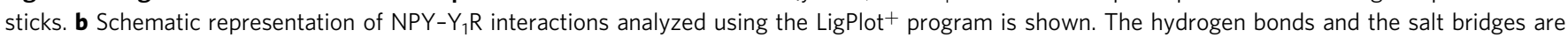
shown in blue and red dashed lines, respectively. c-e Detailed interactions between $\mathrm{C}$-terminal residues of NPY and $\mathrm{Y}_{1} \mathrm{R}$ are shown. The polar contacts are shown as blue dashed lines.

193885-bound inactive $Y_{1} R$ structure shows that $D 287^{6.59}$ of $Y_{1} R$ interacts with the antagonist, whereas $\mathrm{Q}_{2} 19^{5.46}$ of $\mathrm{Y}_{1} \mathrm{R}$ is not involved in this antagonist binding, as Q2195.46 is pushed away by the dihydropyridine group (Fig. $4 \mathrm{~b}$ ). In both inactive structures, Q1203.32 makes van der Waals contact with $\mathrm{M} 310^{7.43}$, although Q120 3.32 rotamers differ in two inactive structures, forming polar interaction with BMS-193885 or van der Waals interaction with W276 6.48 (Fig. 4c). In the NPY-bound $\mathrm{Y}_{1} \mathrm{R}$ structure, the Q120 3.32 sidechain adopts an upward-facing rotamer, forming a polar contact with the amidated C-terminus. Also, Q1203.32 no longer interacts with $\mathrm{M} 310^{7.43}$ (>7 $\mathrm{A}$ ) but with $\mathrm{C} 93^{2.57}$. Reorganization of interaction network near Q120 $0^{3.32}$ by NPY binding stabilizes the conformation of the upward displacement of TM3 (Fig. 4c).

The hydrophobic moieties of the antagonists form hydrophobic networks with $\mathrm{I} 124^{3.36}, \mathrm{I} 128^{3.40}, \mathrm{~F} 272^{6.44}$, W276 $6^{6.48}$, and L2796.51 at the bottom of the ligand-binding pocket and with F282 ${ }^{6.54}$, $\mathrm{F} 286^{6.58}$, and $\mathrm{F} 302^{7.35}$ near the entrance to the binding pocket, to stabilize the inactive $\mathrm{Y}_{1} \mathrm{R}$ structure. These hydrophobic networks are rearranged in the NPY-bound structure. As mentioned above, at the bottom of the ligand-binding pocket, a rotamer change of $\mathrm{I} 128^{3.40}$ and repacking of $\mathrm{F} 272^{6.44}$ and W276 6.48 occur upon NPY binding (Fig. 2b). The three phenylalanine residues $\mathrm{F} 282^{6.54}$, F286 ${ }^{6.58}$, and F302 7.35 on TM6 and TM7 form a stable $\pi-\pi$ network with phenyl groups present in both antagonists; however, this aromatic network is disrupted in the NPY-bound structure. The phenyl ring of F2866.58 flips to form van der Waals contact with L30 and a $\pi$-cation interaction with R33 of NPY (Fig. 4d). F2826.54 and $\mathrm{F} 302^{7.35}$ form a new interaction network with $\mathrm{H} 298^{7.31}$ and I293 ${ }^{\text {ECL3 }}$ (Fig. 4e). F286 $6^{6.58}$ also interacts with Y1 and P2 of NPY. The NPY N-terminus interactions are discussed in the next section.

Comparison of binding modes between the NPY C-terminal tail and antagonists reveals common interactions of the ligand for
$\mathrm{Y}_{1} \mathrm{R}$ binding and NPY-specific interactions, providing hints for designing novel $\mathrm{Y}_{1} \mathrm{R}$ ligands that bind to the $\mathrm{TM}$ core. In addition, the structural comparison of the TM core between inactive and active states of $\mathrm{Y}_{1} \mathrm{R}$ suggests the key events of conformational changes during activation by NPY, rearrangement of the hydrophobic network around F286 ${ }^{6.58}$ at the entrance to the ligand-binding pocket, and rearrangement of interaction network around Q $120^{3.32}$ and $\mathrm{I} 128^{3.40}$ in the connector region at the bottom of the ligand-binding pocket.

Binding of the N-terminal and helical regions of NPY to $Y_{1} R$. In the $\mathrm{OxB}$-bound $\mathrm{OX}_{2} \mathrm{R}$ structure, the $\mathrm{N}$-terminus of $\mathrm{OxB}$ was not observed despite being necessary for signaling. In our complex structure, we were surprised to observe the density of the helical and the N-terminal loop regions of NPY in addition to the C-terminal tail (Fig. 1c). The NPY residues 20-31 were built as an $\alpha$-helix based on the continuous cryo-EM density from the C-terminus (Fig. 1c and Supplementary Fig. 4). This a-helical region forms relatively loose interactions with ECLs compared to the C-terminal tail of NPY that forms extensive interactions with the TM core. The cryoEM map density was weaker toward the N-terminus of the helix, suggesting the flexibility of this region. Three independent $1-\mu \mathrm{s}$ MD simulations using a model composed of full-length NPY, $\mathrm{Y}_{1} \mathrm{R}$ (2-339), and $G_{i 1}$ protein showed that the angle of the helical axis of NPY to the membrane normal varied from $5^{\circ}$ to $70^{\circ}$ during the simulations (Supplementary Fig. 14). While the C-terminal tail of NPY maintained its binding pose during the simulations, the movement increased toward the N-terminus of the NPY helix, explaining the weak density of the N-terminal end of the NPY helix.

The density of side chains in the ECL2 region spanning residues $185-193$ was unclear, but we identified that P183 ${ }^{\mathrm{ECL} 2}$, 
a

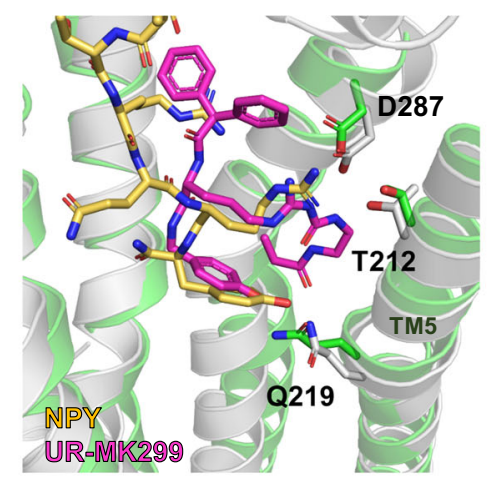

b

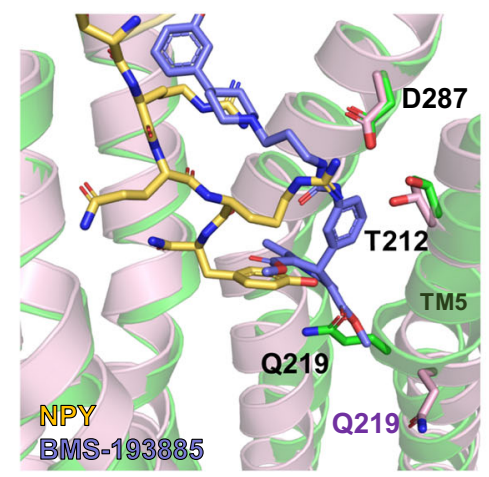

C
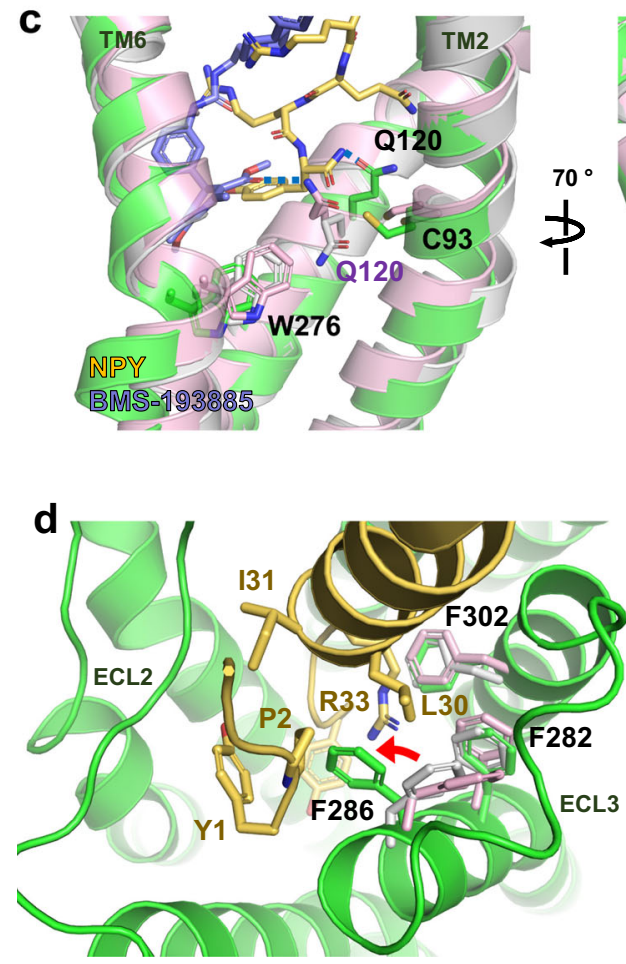

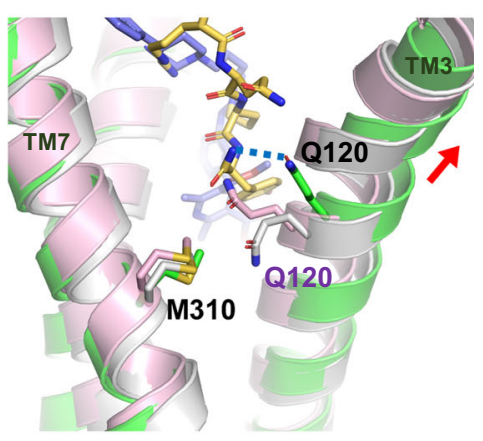

e

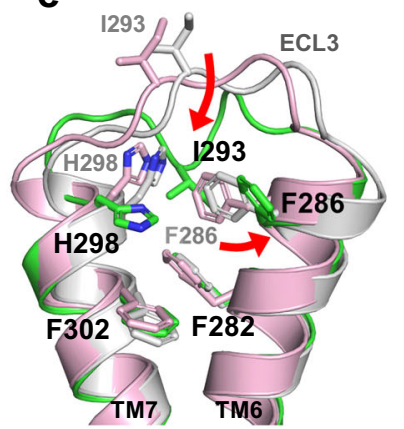

Fig. 4 Comparison of binding mode between NPY and antagonists. a Ligand-binding site occupied by UR-MK299 (PDB ID 5ZBQ, magenta) and $\mathbf{b}$ BMS193885 (PDB ID 5ZBH, slate) is compared with the binding site for NPY (yellow). The side chains of Q2195.46 are differently oriented in all three structures, whereas D2876.59 and T2125.39 maintain similar interactions. c Q1203.32-mediated interactions in the antagonist-bound and NPY-bound structures are compared. In the NPY-bound active structure of $Y_{1} R$, TM3 including Q1203.32 shifts upward and Q1203.32 forms a polar interaction with the C-terminal amide of NPY through its upward-facing sidechain. $\mathbf{d}$ Upon NPY binding, F2866.58 rotamer is changed to form the interactions with NPY R33, L30, and Y1. e Upon NPY binding, I293ECL3 participates in a hydrophobic interaction network with F2866.58, H2987.31, and F3027.35. UR-MK299-bound Y R is indicated in light gray, and BMS-193885-bound $Y_{1} R$ is in pink. Red arrows represent positional changes of $1293^{E C L 3}$ and F286 6.58 upon NPY binding.

F184 ${ }^{\text {ECL2 }}$, and F199ECL2 interacted with Y27, I28, and I31 of NPY, respectively (Fig. 5a). The F184 ${ }^{\mathrm{ECL} 2} \mathrm{~A}$ and F199 ${ }^{\mathrm{ECL} 2} \mathrm{~A}$ mutants reduce the potency of NPY by 38 -fold and 2.3-fold, respectively (Supplementary Table 2 and Supplementary Figs. 15, 16, and 17). Although the F202 ${ }^{\mathrm{ECL} 2} \mathrm{~A}$ mutation exhibited attenuated $\mathrm{G}$ protein signaling, F202 ${ }^{\mathrm{ECL} 2}$ did not interact with NPY. F202 $2^{\text {ECL2 }}$ appears to be important for maintaining the structural integrity of the receptor by forming an aromatic network with $\mathrm{F} 173^{4.60}$, Y176 4.63 , and $\mathrm{Y} 211^{5.38}$, as observed in both inactive and active structures (Supplementary Fig. 18).

Previously, it was proposed that the complete N-terminus of $\mathrm{NPY}$ is necessary for NPY signaling through $\mathrm{Y}_{1} \mathrm{R}^{10}$. Consistently, our signaling assays demonstrated the decrease in the potency of NPY(3-36) and NPY(18-36) by 18-fold and 300-fold, respectively, compared to full-length NPY (Fig. 5b), suggesting that the N-terminal residues of NPY are important for $Y_{1} R$ binding. In our structure, NPY residues 1-5 were modeled by fitting these residues into a cryo-EM map (Fig. 1c and Supplementary Fig. 19). Among these five residues, the $\mathrm{N}$-terminal residue $\mathrm{Y} 1$ was relatively well defined by the cryoEM density. Y1 interacts with F199 ${ }^{\mathrm{ECL} 2}$, D200 ${ }^{\mathrm{ECL} 2}$, and R2085.35 of $Y_{1} R$ (Fig. $5 c$ ), all of which were demonstrated to be important for NPY signaling by previous or current mutational studies ${ }^{27,28}$ (Supplementary Table 2 and Supplementary Figs. 15, 16, and 17).

The N-terminus of NPY does not enter the TM core like the C-terminus but is exposed to the solvent, suggesting its propensity to interact more dynamically with $\mathrm{Y}_{1} \mathrm{R}$. In agreement with our speculation, MD simulations show that the N-terminal 

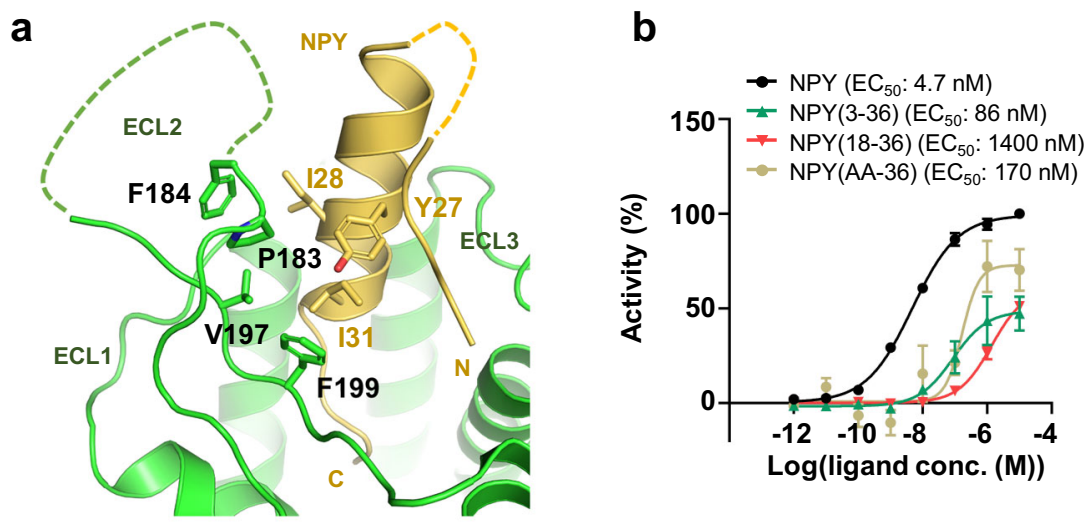

C
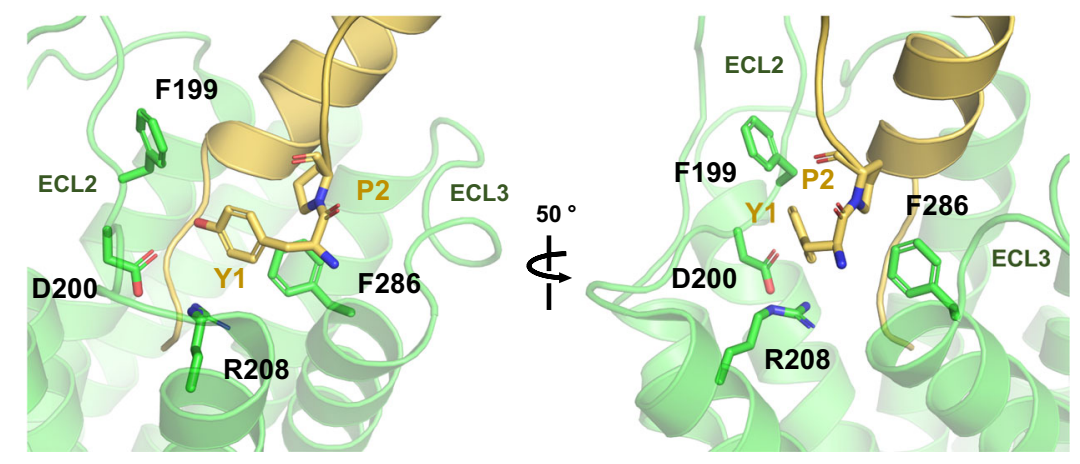

Fig. 5 Binding of NPY $\mathbf{N}$-terminus to $\mathbf{Y}_{\mathbf{1}} \mathbf{R}$. a The sticks show the residues participating in van der Waals interactions between NPY helix and ECL2 of $Y_{1} R$. Unresolved regions of NPY and ECL2 of $Y_{1} R$ are shown with dashed lines. $\mathbf{b}$ The results of $\mathrm{Ca}^{2+}$ assays performed with $\mathrm{N}$-terminally truncated NPY peptides are shown. Removal of two N-terminal residues dramatically reduces NPY potency and efficacy. Symbol and error bar indicate the mean and S.E.M. (standard error of mean) of $n=$ three (NPY(3-36), NPY(18-36), and NPY(AA-36)) or $n=17$ (NPY) independent experiments, respectively. Calculated $\mathrm{EC}_{50}$ values $\left(\mathrm{pEC}_{50} \pm \mathrm{SEM}\right.$ ) are presented on each graph and are summarized in Supplementary Table 2. Source data are provided as a Source Data file. $\mathbf{c}$ Van der Waals interactions of $\mathrm{Y} 1$ and $P 2$ of NPY with $Y_{1} R$ are shown in two different orientations.

region of NPY exhibits relatively higher motions than the C-terminal tail (32-36) during 1- $\mu$ s simulation. Moreover, the Y1-mediated interactions were broken and reformed in one of the replicates during the simulations (Supplementary Fig. 14). Collectively, our data show that the N-terminal region of NPY interacts dynamically with $\mathrm{Y}_{1} \mathrm{R}$. Our model structure represents a possible conformation of the NPY N-terminus, deduced from the cryo-EM map.

Functional role of the $\mathrm{N}$-terminal region of $\mathrm{Y}_{1} \mathbf{R}$. Our cryo-EM map reveals a continuous density from the $\mathrm{N}$-terminal end of TM1 toward the NPY ligand (Supplementary Fig. 6), suggesting an interaction between them. Previously, the N-terminal region of $\mathrm{Y}_{1} \mathrm{R}$, residues 21-32, was crosslinked to NPY in a photocrosslinking experiment ${ }^{10}$. However, the low-resolution map at TM1 and the $\mathrm{N}$-terminal region of $\mathrm{Y}_{1} \mathrm{R}$ prevented us from unambiguously determining whether the $\mathrm{N}$-terminal region of $\mathrm{Y}_{1} \mathrm{R}$ directly interacted with NPY. Through signaling assays and structural analysis, it was previously proven that $\mathrm{N}$-terminal residues were critical for peptide ligand binding and activation of other closely related peptide receptors, namely $\mathrm{OX}_{2} \mathrm{R}$ and $\mathrm{ET}_{\mathrm{B}} \mathrm{R}^{29-32}$.

To validate the importance of the $N$-terminal region of $Y_{1} R$ for NPY signaling, we constructed two N-terminal deletion mutants of $\mathrm{Y}_{1} \mathrm{R}$, one in which the $25 \mathrm{~N}$-terminal residues were deleted $\left(\mathrm{Y}_{1} \mathrm{R} \Delta 25\right)$ and the other in which the $31 \mathrm{~N}$-terminal residues were deleted $\left(\mathrm{Y}_{1} \mathrm{R} \Delta 31\right)$, and performed BRET and $\mathrm{Ca}^{2+}$ assays (Supplementary Table 2 and Supplementary Figs. 20, 21, and 22). $Y_{1} R \Delta 25$ behaves like wild-type $Y_{1} R$ in recruiting $G_{i 1}$ by NPY treatment, whereas $\mathrm{Y}_{1} \mathrm{R} \Delta 31$ showed attenuated response to NPY; this finding suggests that residues 26-31 are involved in NPY binding and thus in $\mathrm{G}_{\mathrm{i} 1}$ recruitment. In particular, the hydrophobic residues $\mathrm{L}_{2} 6^{\mathrm{N}}$ and $\mathrm{F} 28^{\mathrm{N}}$ in $\mathrm{Y}_{1} \mathrm{R}$ were important for NPY signaling, as indicated by the 2.8 -fold increase in $\mathrm{EC}_{50}$ in the $\mathrm{L} 28^{\mathrm{N}} \mathrm{A} / \mathrm{F} 28^{\mathrm{N}} \mathrm{A}$ mutant (Supplementary Table 2 and Supplementary Figs. 20, 21, and 22). MD simulations show that although the $\mathrm{N}$-terminal region of $\mathrm{Y}_{1} \mathrm{R}$ has high mobility, $\mathrm{L} 26^{\mathrm{N}}$ and $\mathrm{F} 28^{\mathrm{N}}$ are within the Ca distance of 9 and $13 \AA$, respectively, from Y21 in the helical region of NPY (Supplementary Fig. 23). Of note, Y21 of NPY is assumed to be a contact point based on the nearby extra cryo-EM density. In addition, the helical region of NPY was demonstrated to interact with ECL2 during the simulations, suggesting that ECL2 and the N-terminal region of $\mathrm{Y}_{1} \mathrm{R}$ form dynamic interactions with NPY by reorienting themselves extensively to accommodate the NPY binding (Supplementary Fig. 23). Thus, we hypothesize that the helical region of NPY forms a tripartite interaction with ECL2 and the $\mathrm{N}$-terminal region of $\mathrm{Y}_{1} \mathrm{R}$, both are shown to partially cover the ligand-binding pocket in the antagonist-bound $\mathrm{Y}_{1} \mathrm{R}$ structures.

\section{Discussion}

Several structures of the G protein-bound active state of class A GPCRs in complex with endogenous peptide agonists, such as NTS (8-13), OxB, and cholecystokinin-8 (CCK-8), have been reported $24,33,34$. Commonly, these peptide agonists have a C-terminal region that inserts into the receptor TM core and acts as a "message" domain (Supplementary Fig. 24) ${ }^{35,36}$. Similarly, in this study, we observed that the five NPY C-terminal residues in 
the NPY-bound $\mathrm{Y}_{1} \mathrm{R}$ structure, forming an extended conformation, make extensive contact with residues in the TM core. In addition, our structure shows that the helical region and the $\mathrm{N}$-terminal loop of NPY interact with $\mathrm{Y}_{1} \mathrm{R}$, although this interaction is much more dynamic and even transient, as indicated by the weaker density for this region in our cryo-EM map, as well as our MD simulations. This study presents a model candidate containing the five N-terminal residues of NPY, constructed based on our cryo-EM map. The cryo-EM density for Y1 of NPY is relatively well resolved, showing interaction with F199ECL2, $\mathrm{D} 200^{\mathrm{ECL} 2}$, and R208 $8^{5.35}$ of $\mathrm{Y}_{1} \mathrm{R}$. Despite being a dynamic interaction, the NPY N-terminus is crucial for $G_{i}$ signaling, as demonstrated by reduced $\mathrm{G}_{\mathrm{i}}$ recruitment in the BRET assay and a 18 -fold increase in $\mathrm{EC}_{50}$ value in signaling assays after treatment with NPY(3-36). The NPY receptor has two other peptide ligands, PYY and PP. PYY is released in response to nutrient intake along the gut and is highly homologous to NPY with $67 \%$ sequence identity; its $\mathrm{N}$-terminus starts with tyrosine, similar to NPY (Supplementary Fig. 25). However, the major circulating form of PYY is the cleaved form PYY(3-36), known to be selective for $\mathrm{Y}_{2} \mathrm{R}^{12,13,37}$. Our signaling assays with NPY, PYY, NPY(3-36), and PYY(3-36) also show that $\mathrm{Y}_{1} \mathrm{R}$ has $\mathrm{EC}_{50}$ values in the nanomolar range for NPY and PYY (4.7 and $6.1 \mathrm{nM}$, respectively) and 13-18-fold increased $\mathrm{EC}_{50}$ values for NPY(3-36) and PYY(3-36) (86 $\mathrm{nM}$ and $77 \mathrm{nM}$, respectively) (Supplementary Fig. 26), suggesting that PYY would bind $Y_{1} R$ similarly to NPY if its N-terminus remains intact. On the contrary, $\mathrm{PP}$ is secreted in the pancreas and has $50 \%$ sequence identity with NPY (Supplementary Fig. 25). Reportedly, PP does not bind $\mathrm{Y}_{1} \mathrm{R}$ at all ${ }^{38}$. PP has $\mathrm{A} 1$ and P34 instead of $\mathrm{Y} 1$ and Q34, respectively; thus, the interactions of $\mathrm{Y} 1$ and Q34 as shown in our NPY-bound $\mathrm{Y}_{1} \mathrm{R}$ structure are important for receptor binding. Indeed, our signaling assay shows that $\mathrm{Y}_{1} \mathrm{R}$ has a 64 -fold increased $\mathrm{EC}_{50}$ value for PP compared to NPY (Supplementary Fig. 26). Among four subtypes of the NPY receptor, $Y_{4} R$ was activated in response to $\mathrm{PP}^{38-40}$. Interestingly, $\mathrm{Y}_{4} \mathrm{R}$ has Glu at the position of $6.58\left(\mathrm{E} 288^{6.58}\right)$, instead of Phe as in Y1R (Supplementary Fig. 27), suggesting that $\mathrm{Y}_{4} \mathrm{R}$ would form a charged interaction network with nearby charged residues (E203ECL2, $\left.\mathrm{R} 211^{5.35}, \mathrm{~T} 215^{5.39}, \mathrm{~N} 285^{6.55}, \mathrm{E} 288^{6.58}, \mathrm{D} 289^{6.59}\right)$ and basic residues of PP, R33 and R35 (Supplementary Fig. 28). We speculate that this extensive charged interaction network would provide sufficient interaction energy for $\mathrm{Y}_{4} \mathrm{R}$ to accommodate $\mathrm{PP}$ as well as NPY, which should be validated with experimental data.

Unlike the previously predicted NPY binding pose, our structure shows that the C-terminal amide of NPY points toward $\mathrm{Q} 120^{3.32}$ of $\mathrm{Y}_{1} \mathrm{R}$, and the $\mathrm{Y} 36$ side chain interacts with Q2195.46. The importance of Q1203.32 and Q2195.46 for NPY binding and signaling is demonstrated by the approximately 2 and 4 -fold increased $\mathrm{EC}_{50}$ values measured using the mutants $\mathrm{Q} 120^{3.32} \mathrm{~A}$ and Q2195.46 L, respectively. Notably, the Y36 side chain occupies a position similar to the hydroxyphenyl ring of the antagonist URMK299 ${ }^{10}$. Thus, in both active and inactive structures, Q2195.46 forms a hydrogen bond with the hydroxyl group of the hydroxyphenyl ring in each ligand. In contrast, Q120 $0^{3.32}$ of $\mathrm{Y}_{1} \mathrm{R}$ forms a hydrogen bond with the C-terminal amide of NPY; however, it is not involved in antagonist binding. Notably, the conserved Gln residue at position 3.32 in $\mathrm{OX}_{2} \mathrm{R}\left(\mathrm{Q} 134^{3.32}\right)$ forms a hydrogen bond with the peptide agonist. Therefore, it was proposed to be a key residue in facilitating the transition to an active state of $\mathrm{OX}_{2} \mathrm{R}$ by a rotamer change to its upward-facing extended conformation $^{24}$. This proposal appears to apply to the $\mathrm{Y}_{1} \mathrm{R}$ activation mechanism, as a similar rotamer change of Q120 $0^{3.32}$ is observed upon activation (Fig. 6).

Comparison of the inactive and active structures suggests the activation mechanism of $\mathrm{Y}_{1} \mathrm{R}$ upon NPY binding. In the published antagonist-bound structures, the three phenylalanine residues $\mathrm{F} 282^{6.54}, \mathrm{~F} 286^{6.58}$, and $\mathrm{F} 302^{7.35}$ located near the entrance to the ligand-binding pocket constitute a hydrophobic cluster with the antagonists UR-MK299 and BMS-193885. Upon NPY binding, R33 of NPY is inserted into this Phe network, causing a rotamer change in F286 6.58 , disrupting the aromatic network. In association with the conformational change of ECL3, a new interaction network including Y1 and R33 of NPY and F282 6.54 , $\mathrm{F} 286^{6.58}, \mathrm{~F} 302^{7.35}, \mathrm{I} 293^{\mathrm{ECL}} 3$, and $\mathrm{H} 298^{7.31}$ of $\mathrm{Y}_{1} \mathrm{R}$ is formed, stabilizing the NPY-bound $\mathrm{Y}_{1} \mathrm{R}$ structure (Fig. 4e). At the bottom of the ligand-binding pocket, Y36 of NPY forms hydrophobic interaction with $\mathrm{I} 124^{3.36}$ through the phenyl group and polar interaction with Q1203.32 through the amidated C-terminus, leading to a rotamer change of Q1203.32. This is followed by a rotamer change of $\mathrm{I} 128^{3.40}$, which interacts with $\mathrm{I} 124^{3.36}$ and repacking of the side chains of $\mathrm{P} 223^{5.50}, \mathrm{~F} 272^{6.44}$, and W276 6.48 . A series of these changes upon NPY binding pulls TM3 upward and causes outward movement of the cytoplasmic region of TM6 (Fig. 6).

One of the key NPY-interacting residues, F286 6.58 , is not conserved and replaced by valine in $\mathrm{Y}_{2} \mathrm{R}$ and glutamate in $\mathrm{Y}_{4} \mathrm{R}$, both of which cannot form $\pi$-cation interaction with $\mathrm{R} 33$ as F286 6.58 does. The difference in the Phe network may explain the $\mathrm{Y}_{1} \mathrm{R}$ selectivity of the two antagonists used to determine the inactive $Y_{1} R$ structures; additionally, it suggests that $Y_{1} R, Y_{2} R$, and $\mathrm{Y}_{4} \mathrm{R}$ may have different interaction networks with NPY. In addition to F286 6.58 , Q2195.46, which forms a hydrogen bond with Y36, is replaced with $\mathrm{L} 227^{5.46}$ in $\mathrm{Y}_{2} \mathrm{R}$, and $\mathrm{H} 298^{7.31}$, which forms an interaction network with F282 6.54 , F286 6.58 , and $\mathrm{F} 302^{7.35}$ is replaced with $\mathrm{G} 300^{7.31}$ in $\mathrm{Y}_{4} \mathrm{R}$. It should also be noted that F $286^{6.58}$ is located within a distance of $4-5 \AA$ to $\mathrm{Y} 1$ and $\mathrm{P} 2$ of NPY. Further details would only be explained by investigating the NPY-bound $\mathrm{Y}_{2} \mathrm{R}$ and $\mathrm{Y}_{4} \mathrm{R}$ structures in the future. However, the vicinity of the residue and the importance of the Phe network suggest that F286 ${ }^{6.58}$ may contribute to the difference between $\mathrm{Y}_{1} \mathrm{R}$ and $\mathrm{Y}_{2} \mathrm{R}$ in the need for the complete $\mathrm{N}$-terminus to elicit a full response.

\section{Methods}

Expression and purification of $\mathbf{Y}_{\mathbf{1}} \mathbf{R}$. Wild-type human $\mathrm{Y}_{1} \mathrm{R}(2-384)$ with a FLAG tag at its $\mathrm{N}$-terminus as well as eGFP and $\mathrm{a} \mathrm{His}_{8}$ tag at its $\mathrm{C}$-terminus, cleavable by HRV 3C protease, was expressed in Spodoptera frugiperda (Sf9) insect cells using the Bac-to-Bac system (Invitrogen). Cells were harvested $48 \mathrm{~h}$ after infection and lysed by repeated dounce homogenization with lysis buffer $(20 \mathrm{mM}$ HEPES pH 8.0, $150 \mathrm{mM} \mathrm{NaCl}$, and $1 \mathrm{mM}$ EDTA) supplemented with phenylmethylsulfonyl fluoride (PMSF), benzamidine, and leupeptin. $\mathrm{Y}_{1} \mathrm{R}$ was extracted from the cell membrane using a solubilization buffer consisting of $20 \mathrm{mM}$ HEPES $\mathrm{pH}$ 8.0, $150 \mathrm{mM} \mathrm{NaCl}, 1 \%(\mathrm{w} / \mathrm{v}) \mathrm{n}$-dodecyl- $\beta$-D-maltoside (DDM), $0.1 \%(\mathrm{w} / \mathrm{v})$ cholesterol hemisuccinate (CHS), PMSF, benzamidine, and leupeptin. After centrifugation, the supernatant was incubated with Ni-NTA resin for $1 \mathrm{~h}$ at $4{ }^{\circ} \mathrm{C}$. After column washing with wash buffer $(20 \mathrm{mM}$ HEPES pH $8.0,150 \mathrm{mM} \mathrm{NaCl}, 20 \mathrm{mM}$ imidazole, $0.05 \%$ DDM, and $0.005 \%$ CHS), the bound protein was eluted with $300 \mathrm{mM}$ imidazole and subsequently loaded onto anti-FLAG M1 agarose resin (Sigma Aldrich) in the presence of $2 \mathrm{mM} \mathrm{CaCl}_{2}$. After column washing and on-column exchange of detergent from DDM to glyco-diosgenin (GDN), the bound protein was eluted with M1 elution buffer (20 mM HEPES pH 8.0, $150 \mathrm{mM} \mathrm{NaCl}, 0.01 \%$ GDN, $0.1 \mathrm{mg} / \mathrm{ml}$ FLAG peptide, and $4 \mathrm{mM}$ EDTA). Heterogeneous glycosylation was removed by PNGase F (NEB), and the eGFP was cleaved by the HRV 3C protease (homemade). $Y_{1} R$ was further purified using a Superdex $20010 / 300$ gel filtration column (Cytiva) pre-equilibrated with a buffer containing $20 \mathrm{mM}$ HEPES $\mathrm{pH} 8.0,150 \mathrm{mM} \mathrm{NaCl}$, and $0.01 \% \mathrm{GDN}$. Freshly purified $\mathrm{Y}_{1} \mathrm{R}$ was used to form a complex with $\mathrm{G}_{\mathrm{i} 1}$ heterotrimer.

Purification of the G protein. Human $\mathrm{Ga}_{\mathrm{i} 1}$ with a $\mathrm{His}_{6}$ tag at its $\mathrm{N}$-terminus was expressed in Escherichia coli (E. coli) Rosetta (DE3) cells. Protein expression was induced with $0.5 \mathrm{mM}$ isopropyl $\beta$-D-1-thiogalactopyranoside (IPTG), and the cells were harvested after incubation at $25^{\circ} \mathrm{C}$ overnight. Cells were lysed with Emulsiflex C3 (Avestin), and the cleared lysate after centrifugation was loaded onto the $\mathrm{Ni}$ NTA column. The column was washed with wash buffer $(20 \mathrm{mM}$ Tris- $\mathrm{HCl} \mathrm{pH} 8.5$, $150 \mathrm{mM} \mathrm{NaCl}, 30 \mathrm{mM}$ imidazole), and the bound protein was eluted with elution buffer (20 mM Tris- $\mathrm{HCl} \mathrm{pH} \mathrm{8.5,} 150 \mathrm{mM} \mathrm{NaCl}$, and $300 \mathrm{mM}$ imidazole). A His 


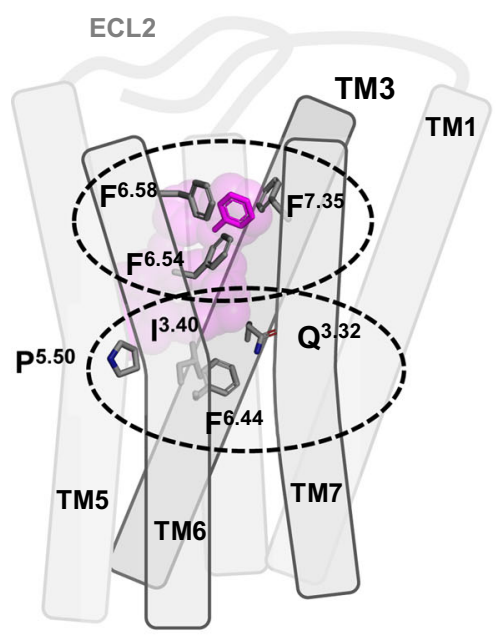

Antagonist-bound

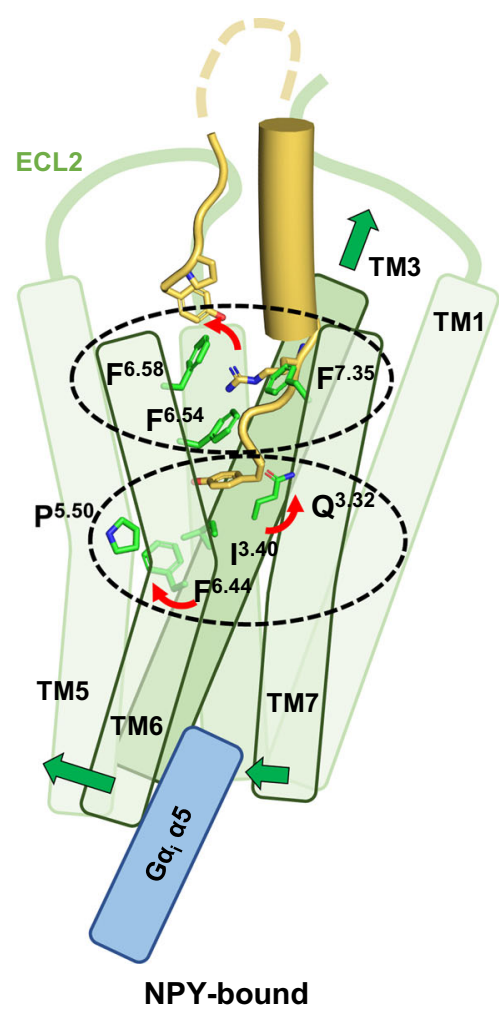

Fig. 6 Mechanism of $\mathbf{Y}_{\mathbf{1}} \mathbf{R}$ activation by NPY binding. Schematic figures representing antagonist-bound (left) and NPY-bound (right) $Y_{1} R$ are shown. Two key regions for $Y_{1} R$ activation are indicated by dashed ellipses. The three phenylalanine residues at TM6 and TM7, and Q1203.32, $1128^{3.40}, P 223^{5.50}$, and F2726.44 in the connector region are shown in stick representation. The three phenylalanine residues participate in antagonist binding through $\pi-\pi$ interactions, but these interactions are rearranged upon NPY binding. F286 6.58 flips and forms a new interaction network with Y1 and R33 of NPY. Since F2866.58 is not conserved in $Y_{2} R$, F2866.58-mediated interactions are $Y_{1} R$-selective. Q1203.32, $1128^{3.40}$ and F2726.44 show rotamer changes upon NPY binding. A series of these changes upon NPY binding pulls TM3 upward and causes outward movement of the cytoplasmic region of TM6.

tag was cleaved by TEV protease (homemade) treatment at $4{ }^{\circ} \mathrm{C}$ overnight. The Hitrap Q column (Cytiva) and a gel filtration column were used for further purification. Purified $\mathrm{Ga}_{\mathrm{i} 1}$ dissolved in a solution composed of $20 \mathrm{mM}$ Tris- $\mathrm{HCl} \mathrm{pH}$ $8.5,50 \mathrm{mM} \mathrm{NaCl}, 1 \mathrm{mM} \mathrm{MgCl}_{2}$, and $10 \mu \mathrm{M}$ guanosine diphosphate (GDP) was concentrated, snap frozen in liquid nitrogen, and stored at $-80^{\circ} \mathrm{C}$ until use.

Human $\mathrm{G} \beta_{1}$ with a $\mathrm{His}_{6}$ tag at its $\mathrm{N}$-terminus and $\mathrm{G} \gamma_{2}$ with a $\mathrm{C} 68 \mathrm{~S}$ mutation were co-expressed in Sf9 insect cells. Cells were harvested $72 \mathrm{~h}$ after incubation and lysed with lysis buffer ( $20 \mathrm{mM}$ Tris- $\mathrm{HCl} \mathrm{pH} \mathrm{8.5,} 0.1 \mathrm{mM}$ Tris (2-carboxyethyl) phosphine hydrochloride (TCEP), and protease inhibitors). After centrifugation, the cleared supernatant was loaded onto an Ni-NTA column. The bound protein was eluted with lysis buffer supplemented with $300 \mathrm{mM}$ imidazole after washing with lysis buffer supplemented with $20 \mathrm{mM}$ imidazole. The $\mathrm{His}_{6}$ tag was cleaved with HRV $3 \mathrm{C}$ protease, and the $\mathrm{G} \beta \gamma$ complex was further purified using a Hitrap Q column.

For $\mathrm{G}$ protein heterotrimer formation, purified $\mathrm{Ga}_{\mathrm{i} 1}$ and $\mathrm{G} \beta \gamma$ were mixed in a 1.1:1 molar ratio with excess $\mathrm{MgCl}_{2}$ and GDP. After an hour of incubation on ice, the $\mathrm{Ga}_{i} \beta \gamma$ complex was purified using a gel filtration column equilibrated with a solution composed of $20 \mathrm{mM}$ HEPES pH 7.5, $150 \mathrm{mM} \mathrm{NaCl}, 1 \mathrm{mM} \mathrm{MgCl}$, and $10 \mu \mathrm{M}$ GDP. The purified $\mathrm{Ga}_{\mathrm{i}} \beta \gamma$ complex was concentrated, snap frozen in liquid nitrogen, and stored at $-80^{\circ} \mathrm{C}$ until use.

We confirmed that $\mathrm{Ga}_{\mathrm{i} 1}$ produced from E. coli is still functional using GTP turnover assay (Supplementary Fig. 29). For comparison, we purified $\mathrm{G}_{\mathrm{i} 1}$ heterotrimer produced from Sf9 insect cells, as previously described ${ }^{22}$

Purification of the scFv16. The scFv16 construct was kindly provided by Dr. Kobilka (Stanford University). Purification of scFv16 was performed by the previously described method with slight modifications ${ }^{19}$. First, scFv16 with a His 6 tag at its C-terminus was expressed in Trichoplusia ni (Hi5) cells. After cell harvesting, the supernatant containing secreted scFv16 was incubated with Ni-NTA resin at $4{ }^{\circ} \mathrm{C}$ for $2 \mathrm{~h}$. The resin was washed with buffer $(20 \mathrm{mM}$ HEPES $\mathrm{pH} 7.0$, and $150 \mathrm{mM} \mathrm{NaCl}, 30 \mathrm{mM}$ imidazole), and the bound protein was eluted with the buffer supplemented with an additional $300 \mathrm{mM}$ imidazole. Further purification was performed using a gel filtration column pre-equilibrated with a solution composed of $20 \mathrm{mM}$ HEPES pH 7.0, and $150 \mathrm{mM} \mathrm{NaCl}$. Purified scFv16 was concentrated, snap frozen in liquid nitrogen, and stored at $-80^{\circ} \mathrm{C}$ until use.
Purification of the NPY-Y $\mathbf{R}_{\mathbf{1}} \mathbf{-}-\mathbf{G}_{\mathbf{i 1}}$-scFv16 complex. Purified $\mathrm{Y}_{1} \mathrm{R}, \mathrm{G}_{\mathrm{i} 1}$ heterotrimer, and scFv16 were mixed at a molar ratio of 1:1.1:1.2 in the presence of excess NPY (GL Biochem, Shanghai, China) and incubated overnight at $4{ }^{\circ} \mathrm{C}$ with apyrase (NEB, MA, USA). The sample was then loaded onto a gel filtration column preequilibrated with a solution composed of $20 \mathrm{mM}$ HEPES pH $8.0,150 \mathrm{mM} \mathrm{NaCl}$, $0.01 \% \mathrm{GDN}$, and $2 \mu \mathrm{M}$ NPY. The purified NPY $-\mathrm{Y}_{1} \mathrm{R}-\mathrm{G}_{\mathrm{i} 1}-\mathrm{scFv} 16$ complex was concentrated to $10 \mathrm{mg} / \mathrm{ml}$ and used for cryo-EM grid preparation.

Cryo-EM grid preparation and data collection. An aliquot (3.5 $\mu \mathrm{l})$ of purified $\mathrm{NPY}-\mathrm{Y}_{1} \mathrm{R}-\mathrm{G}_{11}-\mathrm{scFv} 16$ complex was applied onto a glow-discharged holey carbon grid (Quantifoil R1.2/1.3, 300 mesh). The grids were blotted for $5 \mathrm{~s}$ at $12^{\circ} \mathrm{C}$ and $100 \%$ humidity and plunge-frozen in liquid ethane using a Vitrobot Mark IV (Thermo Fisher Scientific, USA) at Center for Macromolecular and Cell imaging of Seoul National University (SNU CMCI). Grids were initially screened with the FEI Glacios (Thermo Fisher Scientific, USA) at SNU CMCI, equipped with a Falcon 4 detector. Images were acquired on a 300-kV Titan Krios (Thermo Fisher Scientific, USA) at Korea Basic Science Institute, equipped with a Falcon 3EC direct electron detector. Movies were recorded in counting mode at a magnification of $\times 161,850$ (corresponding to a calibrated pixel size of $0.865 \AA$ ) and a defocus range of -1.25 to $-2.75 \mu \mathrm{m}$. A total of 4965 movies were collected, each comprising 40 frames, with a total dose of 40 electrons per $\AA^{2}$. A detailed description of the cryo-EM data collection parameters is provided in Supplementary Table 1.

Three-dimensional (3D) reconstruction of NPY-Y,R-G $\mathrm{G}_{\mathrm{i1}}-\mathrm{ScFv16}$ complex. Image stack preprocessing was performed using CryoSPARC v. 3.1 (Structura Biotechnology ${ }^{41}$. All movies subjected to beam-induced motion correction using patched-motion correction and contrast transfer function (CTF) parameters for each non-dose-weighted micrograph were determined by patch CTF estimation. After initial particle picking and two-dimensional classification, selected good particles were used for Topaz training 42 . Topaz picking particles $(1,300,000$ particles) were extracted and subjected to three rounds of heterogeneous refinement Further heterogeneity classifications were performed by 3D-variability analysis (3DVA) ${ }^{43}$, focusing on the NPY $-Y_{1} R-G_{i 1}$ complex without micelles, $G \alpha_{i}$ AHD, and scFv16 by masking. Clusters with well-resolved density were obtained and used for final map reconstruction by non-uniform refinement ${ }^{44}$. Maps for this 
processing have a global nominal resolution of $3.2 \AA$, based on gold-standard Fourier shell correlation using the 0.143 criteria. To improve the map quality of $\mathrm{NPY}-\mathrm{Y}_{1} \mathrm{R}-\mathrm{G}_{\mathrm{i1}}$ complex, we performed the local refinement focusing on the NPY- $Y_{1} R$ and $G_{i 1}-s c F v 16$ in cryoSPARC v3.2. These local refinements generated the maps at a global nominal resolution of $3.6 \AA$ and $3.1 \AA$, respectively. Cryo-EM density for ECL2 (176-183, 194-203) and NPY (1-5, 20-36) was clearly observed in the refined map with a mask on NPY-Y ${ }_{1} R$. These two local refined maps were combined using "vop maximum" command in UCSF chimera to represent and analyze the NPY $-Y_{1} R-G_{i 1}$ complex ${ }^{45}$. The combined map is represented in Fig. 1a. The local resolution was determined using the cryoSPARC local resolution estimation. Local sharpening was performed by LocSpiral to trace the $\mathrm{Y}_{1} \mathrm{R} N$-terminus (beyond L $35^{\mathrm{N}}$ ) and ECL2 region (Supplementary Fig. 6) ${ }^{46}$. No artifacts were observed when compared to the global sharpening map in the other regions.

Model building and refinement. The initial model was obtained by rigid-bodyfitting of the structure of inactive $\mathrm{Y}_{1} \mathrm{R}$ (PDB ID $\left.5 \mathrm{ZBH}\right)^{10}$ and $\mathrm{Ga}_{\mathrm{i}} \beta \gamma$ and $\mathrm{scFv} 16$ from the NTSR1 complex structure (PDB ID 6OS9) ${ }^{22}$. This initial model was then subjected to iterative rounds of manual rebuilding with COOT and refinement with PHENIX ${ }^{47,48}$. The geometry of the refined structure was evaluated using the MolProbity ${ }^{49}$. The final model consisting of NPY, $Y_{1} R, G a_{i}, G \beta, G \gamma$, and scFv16 was deposited in the PDB with PDB code 7VGX, and the electron density map was deposited in the EMDB with ID EMD-31979. The refinement statistics are presented in Supplementary Table 1. All molecular graphic figures were prepared using the UCSF Chimera, UCSF ChimeraX, and PyMol v2.4.045,50,51.

GTP turnover assay. GTP turnover assay was performed using GTPase-Glo assay kit (Promega) ${ }^{22}$. Purified $\mathrm{Y}_{1} \mathrm{R}$ was incubated with NPY for $40 \mathrm{~min}$ at room temperature. NPY-bound $\mathrm{Y}_{1} \mathrm{R}(4 \mu \mathrm{M})$ was mixed with $1 \mu \mathrm{M} \mathrm{G}_{\mathrm{i} 1}$ heterotrimer (containing $\mathrm{Ga}_{\mathrm{i}}$ produced from $E$. coli or Sf9 cells) in an assay buffer consisting of $20 \mathrm{mM}$ HEPES pH 8.0, $150 \mathrm{mM} \mathrm{NaCl}, 0.03 \%$ DDM, $0.003 \%$ CHS, $100 \mu \mathrm{M}$ TCEP, $5 \mu \mathrm{M}$ GDP, and $2.5 \mu \mathrm{M}$ GTP. After incubation for $3 \mathrm{~h}$, reconstituted GTPase-Glo reagent was added to the sample and incubated for $30 \mathrm{~min}$ at room temperature. Then, detection reagent was mixed and incubated for $10 \mathrm{~min}$ at room temperature. Luminescence was measured using a FlexStation 3 multi-mode microplate reader (Molecular Devices) and data were analyzed by GraphPad Prism 9.2.0.

ELISA-based surface expression assay. HEK293T cells were transfected with each expression plasmid of $Y_{1} R$ mutants and wild-type. After $48 \mathrm{~h}$ of incubation, $4 \%$ paraformaldehyde (PFA, T\&I) was treated for fixation and washed with $1 \times$ PBS. After $30 \mathrm{~min}$ of incubation with blocking solution (2.5\% bovine serum albumin, Bovogen), rabbit anti-FLAG antibody (Cell signaling Technology, 1:1000 dilution) was then treated for staining and anti-rabbit HRP antibody (Enzo Life Sciences, 1:1000 dilution) was used for detection. After incubation with HRP antibody, TMB solution (Thermofisher scientific) was added to each well, incubated until blue color was observed. Further reaction was blocked by adding $2 \mathrm{M}$ $\mathrm{HCl}$. The absorbance was detected at $450 \mathrm{~nm}$ with FlexStation 3 multi-mode microplate reader. Normalization was carried out by removing TMB substrate solution from the wells and adding Janus Green solution $(0.2 \% \mathrm{w} / \mathrm{v}$, TCL). Further elimination of excess stain was done by washing with milli $\mathrm{Q}$ water and adding $0.5 \mathrm{M} \mathrm{HCl}$. Absorbance was read at $595 \mathrm{~nm}$. Normalized expression level of the receptor at the cell surface was calculated by the ratio of the absorbance at 450 and $595 \mathrm{~nm}\left(A_{450} / A_{595}\right)$. The graphs were plotted using GraphPad Prism 9.2.0.

BRET assay. HEK293T cells were co-transfected with $\mathrm{Ga}_{\mathrm{i}}$-Rluc, $\mathrm{G} \beta, \mathrm{G} \gamma$, and $\mathrm{Y}_{1} \mathrm{R}$-eYFP constructs at a 1:1:1:5 ratio. Forty-eight hours of post-transfection, cells were detached with PBS supplemented with $20 \mathrm{mM}$ EDTA and evenly spread into white 96-well microplates (SPL). For ligand-induced conditions, various concentrations of NPY were incubated with each $Y_{1} R$ mutant-transfected group, and Coelenterazine $\mathrm{h}$ was added to each well to a final concentration of $5 \mu \mathrm{M}$. All BRET data were collected using a Mithras LB940 instrument (Berthold), and graphs were plotted using GraphPad Prism 9.2.0.

$\mathrm{Ca}^{2+}$ signaling assay. For the ligand-induced $\mathrm{Ca}^{2+}$ assay, HEK293T cells were seeded on 96-well black wall/clear bottom microplate (SPL) in Dulbecco's modified Eagle's medium (Biowest) containing 10\% fetal bovine serum (Biowest) and antibiotic-antimycotic (Gibco) $24 \mathrm{~h}$ before plasmid transfection. After transfection with the plasmid containing $Y_{1} R$ constructs, $G \alpha_{\Delta 6 q i 4 m y r}{ }_{2} G \beta$, and $G \gamma$ in a $3: 1: 1: 1$ ratio, followed by $48 \mathrm{~h}$ incubation, cells were stained with Cal-520 (AAT Bioquest, Inc.) in assay buffer (HBSS, 0.1\% BSA, $20 \mathrm{mM}$ HEPES pH 7.4). After $2 \mathrm{~h}$, Cal-520stained cells were washed three times with assay buffer. Intracellular $\mathrm{Ca}^{2+}$ influx was measured at $\mathrm{Ex} / \mathrm{Em}=490 / 525 \mathrm{~nm}$ using the FlexStation 3 multi-mode microplate reader (Molecular Devices). After $30 \mathrm{~s}$ of baseline, the ligand was injected to achieve the final concentration. Log (concentration)-response curves, used to estimate $\mathrm{EC}_{50}$, were calculated using GraphPad Prism 9.2.0, by fitting an agonist response curve with a variant slope to the normalized response data.

Calculation of the ligand-binding pocket volume. First, to define the interior of the TM bundle, the receptor structure was aligned along the $z$-axis by superimposing a pre-aligned GPCR structure from the OPM database ${ }^{53}$. Thereafter, a 3D grid was constructed with equispaced points covering the receptor structure. The centers of the TM helices for each discrete $z$-axis value and the lines connecting the neighboring centers were defined as the lateral boundaries. For helices without a defined center point for a given $z$-axis value, the $(x, y)$ coordinate of the nearest point was used instead. Next, the upper and lower boundaries of the ligand-binding pocket were defined. The $z$-axis coordinate of the $\mathrm{C} \alpha$ atom of $\mathrm{W}^{6.48}$, a toggle switch residue, was defined as the lower boundary. TM residues closest to the extracellular region were used to define the upper boundary of the pocket volume. Finally, after removing the grid points causing clashes with protein atoms, the cavity volume was calculated from the number of grid points inside the defined boundaries. The python code for calculating the solvent accessible volume of the ligand-binding pocket is available at https://github.com/seoklab/GPCR_binding_cavity_volume_calculation.

$\mathbf{Y}_{\mathbf{4}} \mathbf{R}$-PP homology modeling. The homology model of PP-bound $\mathrm{Y}_{4} \mathrm{R}$ was prepared by template-based modeling protocol, GalaxyTBM, using the current NPYbound $\mathrm{Y}_{1} \mathrm{R}$ structure as a template ${ }^{54}$. The initial model was constructed by threading the target sequence on the template structure, followed by energy optimization and additional structure sampling for unreliable local regions. Physicsbased optimization method GalaxyRefineComplex refined template-based models ${ }^{55}$. A scoring function optimized for GPCR structure prediction in Galaxy7TM was used ${ }^{56}$.

MD simulation. Missing residues were added to the structure model of the NPY $-Y_{1} R-G_{i 1}$ complex to build an initial model for MD simulation. The AHD domain of $\mathrm{G}_{i 1}$ was added by aligning the previously reported structure ${ }^{57}$, and the missing residues of $Y_{1} R$ in the N-terminus (25-34), ECL2 (184-193), ICL2 (247-252), and C-terminus (330-339) were built based on the map with a mask on NPY $-\mathrm{Y}_{1} \mathrm{R}$ at a lower threshold. The rest of the N-terminus (2-24) was extended randomly in a position that did not collide with the existing structure.

This study used the CHARMM36(m) force field for proteins and lipids ${ }^{58-60}$. The TIP3P water model was utilized along with $0.15 \mathrm{M} \mathrm{NaCl}$ solution ${ }^{61}$. Three independent MD simulations were performed for each system to obtain better sampling and check the convergence. Periodic boundary conditions (PBCs) were employed in all simulations. The van der Waals interactions were smoothly switched off over 10-12 A by a force-based switching function and the long-range electrostatic interactions were calculated using the particle-mesh Ewald method with a mesh size of $\sim 1 \AA^{62}$. All simulations were performed using the inputs generated by CHARMM-GUI and GROMACS 2018.6 for both equilibration and production with the LINCS algorithm ${ }^{63-67}$. The temperature was maintained using a Nosé-Hoover temperature coupling method with a $\tau_{t}$ of $1 \mathrm{ps}^{68}$. For pressure coupling ( 1 bar), the semi-isotropic Parrinello-Rahman method with a $\tau_{\mathrm{p}}$ of $5 \mathrm{ps}$ and compressibility of $4.5 \times 10^{-5} \mathrm{bar}^{-1}$ was used ${ }^{69}$. The constant particle number volume, and temperature (NVT) dynamics were first applied with a 1-fs time step for $250 \mathrm{ps}$ during the equilibration run. Subsequently, the constant particle number, pressure, and temperature (NPT) ensemble was applied with a $1 \mathrm{fs}$ time step (for $2 \mathrm{~ns}$ ) and with a 2 fs time step (for $18 \mathrm{~ns}$ ). During the equilibration, positional and dihedral restraint potentials were applied, and their force constants were gradually reduced. The production run was performed with a $4 \mathrm{fs}$ time step using the hydrogen mass repartitioning technique without any restraint potential ${ }^{70}$ Each system ran about $25 \mathrm{~ns} /$ day with $512 \mathrm{CPU}$ cores on NURION in the Korea Institute of Science and Technology Information.

Reporting summary. Further information on research design is available in the Nature Research Reporting Summary linked to this article.

\section{Data availability}

Additional data supporting the findings of this work are available as the Supplementary Information, Supplementary Data and Source Data files. The atomic model has been deposited in the Protein Data Bank under accession code 7VGX and the cryo-EM density maps have been deposited in the Electron Microscopy Data Bank under accession code EMD-31979. The structure of inactive $\mathrm{Y}_{1} \mathrm{R}$ (PDB ID 5ZBH), Ga $\alpha_{i 1} \beta \gamma$ and scFv16 from the NTSR1 complex structure (PDB ID 6OS9) were used as an initial template to build the $\mathrm{NPY}-\mathrm{Y}_{1} \mathrm{R}-\mathrm{G}_{\mathrm{i}}$ protein model. Structural models used in data analysis were accessed from the Protein Data Bank under the accession codes 5ZBQ (UR-MK299 and $\mathrm{Y}_{1} \mathrm{R}$ ), 6DDE $\left(\mu \mathrm{OR}, \mathrm{G}_{\mathrm{i}}\right), 7 \mathrm{~L} 1 \mathrm{U}\left(\mathrm{OxB}\right.$ and $\left.\mathrm{OX}_{2} \mathrm{R}\right), 5 \mathrm{GLH}\left(\mathrm{ET} 1\right.$ and $\left.\mathrm{ET}_{\mathrm{B}} \mathrm{R}\right), 7 \mathrm{~L} 0 \mathrm{Q}$ (NTS and NTSR1), and $7 \mathrm{MBX}$ (CCK-8 and CCK1R). Source data are provided with this paper.

\section{Code availability}

The python code for calculating the solvent accessible volume of the ligand-binding pocket is available at https://github.com/seoklab/GPCR_binding_cavity_volume_calculation and as a Source Data file.

Received: 2 October 2021; Accepted: 26 January 2022; Published online: 14 February 2022 


\section{References}

1. Michel, M. C. et al. XVI. International Union of Pharmacology recommendations for the nomenclature of neuropeptide $\mathrm{Y}$, peptide YY, and pancreatic polypeptide receptors. Pharm. Rev. 50, 143-150 (1998).

2. Herzog, $\mathrm{H}$. et al. Cloned human neuropeptide $\mathrm{Y}$ receptor couples to two different second messenger systems. Proc. Natl Acad. Sci. USA 89, 5794-5798 (1992).

3. Kohno, D. \& Yada, T. Arcuate NPY neurons sense and integrate peripheral metabolic signals to control feeding. Neuropeptides 46, 315-319 (2012).

4. Tasan, R. O. et al. The central and basolateral amygdala are critical sites of neuropeptide $\mathrm{Y} / \mathrm{Y} 2$ receptor-mediated regulation of anxiety and depression. J. Neurosci. 30, 6282-6290 (2010).

5. Gotzsche, C. R. \& Woldbye, D. P. The role of NPY in learning and memory. Neuropeptides 55, 79-89 (2016).

6. Vahatalo, L. H., Ruohonen, S. T., Ailanen, L. \& Savontaus, E. Neuropeptide Y in noradrenergic neurons induces obesity in transgenic mouse models. Neuropeptides 55, 31-37 (2016).

7. Farzi, A., Hassan, A. M., Zenz, G. \& Holzer, P. Diabesity and mood disorders: Multiple links through the microbiota-gut-brain axis. Mol. Asp. Med 66, 80-93 (2019).

8. Zhang, L., Bijker, M. S. \& Herzog, H. The neuropeptide Y system: pathophysiological and therapeutic implications in obesity and cancer. Pharm. Ther. 131, 91-113 (2011).

9. Monks, S. A., Karagianis, G., Howlett, G. J. \& Norton, R. S. Solution structure of human neuropeptide Y. J. Biomol. NMR 8, 379-390 (1996).

10. Yang, Z. et al. Structural basis of ligand binding modes at the neuropeptide $Y$ Y(1) receptor. Nature 556, 520-524 (2018).

11. Beck-Sickinger, A. G. \& Jung, G. Structure-activity relationships of neuropeptide $\mathrm{Y}$ analogues with respect to $\mathrm{Y} 1$ and $\mathrm{Y} 2$ receptors. Biopolymers 37, 123-142 (1995).

12. Mentlein, R., Dahms, P., Grandt, D. \& Kruger, R. Proteolytic processing of neuropeptide Y and peptide YY by dipeptidyl peptidase IV. Regul. Pept. 49, 133-144 (1993).

13. Grandt, D. et al. Novel generation of hormone receptor specificity by amino terminal processing of peptide YY. Biochem Biophys. Res. Commun. 186, 1299-1306 (1992).

14. Wieland, H. A., Willim, K. \& Doods, H. N. Receptor binding profiles of NPY analogues and fragments in different tissues and cell lines. Peptides 16, 1389-1394 (1995).

15. Zheng, H. et al. High-fat intake induced by mu-opioid activation of the nucleus accumbens is inhibited by Y1R-blockade and MC3/4R- stimulation. Brain Res. 1350, 131-138 (2010).

16. Yulyaningsih, E., Zhang, L., Herzog, H. \& Sainsbury, A. NPY receptors as potential targets for anti-obesity drug development. Br. J. Pharm. 163, 1170-1202 (2011).

17. Bhisikar, S. M., Kokare, D. M., Nakhate, K. T., Chopde, C. T. \& Subhedar, N. $\mathrm{K}$. Tolerance to ethanol sedation and withdrawal hyper-excitability is mediated via neuropeptide Y Y1 and Y5 receptors. Life Sci. 85, 765-772 (2009).

18. Liu, L. et al. NPY1R is a novel peripheral blood marker predictive of metastasis and prognosis in breast cancer patients. Oncol. Lett. 9, 891-896 (2015).

19. Maeda, S. et al. Development of an antibody fragment that stabilizes GPCR/Gprotein complexes. Nat. Commun. 9, 3712 (2018).

20. Dong, M. et al. Structure and dynamics of the active Gs-coupled human secretin receptor. Nat. Commun. 11, 4137 (2020).

21. Ballesteros, J. A. \& Weinstein, H. Integrated methods for the construction of three-dimensional models and computational probing of structure-function relations in G protein-coupled receptors. Methods Neurosci. 25, 366-428 (1995).

22. Kato, H. E. et al. Conformational transitions of a neurotensin receptor 1-G(i1) complex. Nature 572, 80-85 (2019).

23. Koehl, A. et al. Structure of the $\mu$-opioid receptor-G(i) protein complex. Nature 558, 547-552 (2018).

24. Hong, C. et al. Structures of active-state orexin receptor 2 rationalize peptide and small-molecule agonist recognition and receptor activation. Nat. Commun. 12, 815 (2021)

25. Lee, J. H. et al. Solution structure of a new hypothalamic neuropeptide, human hypocretin-2/orexin-B. Eur. J. Biochem. 266, 831-839 (1999).

26. Beck-Sickinger, A. G. et al. Complete L-alanine scan of neuropeptide Y reveals ligands binding to Y1 and Y2 receptors with distinguished conformations. Eur. J. Biochem. 225, 947-958 (1994).

27. Sautel, M. et al. Neuropeptide $Y$ and the nonpeptide antagonist BIBP 3226 share an overlapping binding site at the human Y1 receptor. Mol. Pharm. 50, 285-292 (1996).

28. Sjodin, P. et al. Re-evaluation of receptor-ligand interactions of the human neuropeptide Y receptor Y1: a site-directed mutagenesis study. Biochem J. 393, 161-169 (2006).
29. Yin, J. et al. Structure and ligand-binding mechanism of the human OX1 and OX2 orexin receptors. Nat. Struct. Mol. Biol. 23, 293-299 (2016).

30. Rappas, M. et al. Comparison of Orexin 1 and Orexin 2 ligand binding modes using X-ray crystallography and computational analysis. J. Med Chem. 63, 1528-1543 (2020)

31. Takasuka, T., Sakurai, T., Goto, K., Furuichi, Y. \& Watanabe, T. Human endothelin receptor ETB. Amino acid sequence requirements for super stable complex formation with its ligand. J. Biol. Chem. 269, 7509-7513 (1994).

32. Shihoya, W. et al. Crystal structures of human ET(B) receptor provide mechanistic insight into receptor activation and partial activation. Nat. Commun. 9, 4711 (2018).

33. Zhang, M. et al. Cryo-EM structure of an activated GPCR-G protein complex in lipid nanodiscs. Nat. Struct. Mol. Biol. 28, 258-267 (2021).

34. Mobbs, J. I. et al. Structures of the human cholecystokinin 1 (CCK1) receptor bound to $\mathrm{Gs}$ and $\mathrm{Gq}$ mimetic proteins provide insight into mechanisms of $\mathrm{G}$ protein selectivity. PLoS Biol. 19, e3001295 (2021).

35. Schwyzer, R. ACTH: a short introductory review. Ann. N. Y Acad. Sci. 297, 3-26 (1977).

36. Portoghese, P. S. Bivalent ligands and the message-address concept in the design of selective opioid receptor antagonists. Trends Pharm. Sci. 10, 230-235 (1989).

37. Stadlbauer, U., Woods, S. C., Langhans, W. \& Meyer, U. PYY3-36: Beyond food intake. Front. Neuroendocrinol. 38, 1-11 (2015).

38. Cabrele, C., Wieland, H. A., Langer, M., Stidsen, C. E. \& Beck-Sickinger, A. G Y-receptor affinity modulation by the design of pancreatic polypeptide/ neuropeptide $\mathrm{Y}$ chimera led to $\mathrm{Y}(5)$-receptor ligands with picomolar affinity. Peptides 22, 365-378 (2001).

39. Lundell, I. et al. Cloning of a human receptor of the NPY receptor family with high affinity for pancreatic polypeptide and peptide YY. J. Biol. Chem. 270, 29123-29128 (1995).

40. Lin, S. et al. Critical role of arcuate Y4 receptors and the melanocortin system in pancreatic polypeptide-induced reduction in food intake in mice. PLoS ONE 4, e8488 (2009).

41. Punjani, A., Rubinstein, J. L., Fleet, D. J. \& Brubaker, M. A. cryoSPARC algorithms for rapid unsupervised cryo-EM structure determination. Nat. Methods 14, 290-296 (2017).

42. Bepler, T. et al. Positive-unlabeled convolutional neural networks for particle picking in cryo-electron micrographs. Nat. Methods 16, 1153-1160 (2019).

43. Punjani, A. \& Fleet, D. J. 3D variability analysis: resolving continuous flexibility and discrete heterogeneity from single particle cryo-EM. J. Struct. Biol. 213, 107702 (2021).

44. Punjani, A., Zhang, H. \& Fleet, D. J. Non-uniform refinement: adaptive regularization improves single-particle cryo-EM reconstruction. Nat. Methods 17, 1214-1221 (2020).

45. Pettersen, E. F. et al. UCSF Chimera-a visualization system for exploratory research and analysis. J. Comput Chem. 25, 1605-1612 (2004).

46. Kaur, S. et al. Local computational methods to improve the interpretability and analysis of cryo-EM maps. Nat. Commun. 12, 1240 (2021).

47. Emsley, P., Lohkamp, B., Scott, W. G. \& Cowtan, K. Features and development of Coot. Acta Crystallogr D 66, 486-501 (2010)

48. Liebschner, D. et al. Macromolecular structure determination using X-rays, neutrons and electrons: recent developments in Phenix. Acta Crystallogr D 75 861-877 (2019).

49. Williams, C. J. et al. MolProbity: more and better reference data for improved all-atom structure validation. Protein Sci. 27, 293-315 (2018).

50. Goddard, T. D. et al. UCSF ChimeraX: meeting modern challenges in visualization and analysis. Protein Sci. 27, 14-25 (2018).

51. Schrödinger. The PyMOL Molecular Graphics System, Version 2.4.0, Schrödinger LLC. (2020).

52. Kostenis, E. Potentiation of GPCR-signaling via membrane targeting of G protein alpha subunits. J. Recept Signal Transduct. Res. 22, 267-281 (2002).

53. Lomize, M. A., Lomize, A. L., Pogozheva, I. D. \& Mosberg, H. I. OPM: orientations of proteins in membranes database. Bioinformatics 22, 623-625 (2006).

54. Ko, J., Park, H. \& Seok, C. GalaxyTBM: template-based modeling by building a reliable core and refining unreliable local regions. BMC Bioinform. 13, 198 (2012).

55. Heo, L., Lee, H. \& Seok, C. GalaxyRefineComplex: refinement of proteinprotein complex model structures driven by interface repacking. Sci. Rep. 6 32153 (2016).

56. Lee, G. R. \& Seok, C. Galaxy7TM: flexible GPCR-ligand docking by structure refinement. Nucleic Acids Res. 44, W502-W506 (2016).

57. Kang, Y. et al. Cryo-EM structure of human rhodopsin bound to an inhibitory G protein. Nature 558, 553-558 (2018).

58. Huang, J. et al. CHARMM36m: an improved force field for folded and intrinsically disordered proteins. Nat. Methods 14, 71-73 (2017). 
59. Klauda, J. B., Monje, V., Kim, T. \& Im, W. Improving the CHARMM force field for polyunsaturated fatty acid chains. J. Phys. Chem. B 116, 9424-9431 (2012).

60. Klauda, J. B. et al. Update of the CHARMM all-atom additive force field for lipids: validation on six lipid types. J. Phys. Chem. B 114, 7830-7843 (2010).

61. Jorgensen, W. L., Chandrasekhar, J., Madura, J. D., Impey, R. W. \& Klein, M. L. Comparison of simple potential functions for simulating liquid water. $J$. Chem. Phys. 79, 926-935 (1983).

62. Essmann, U. et al. A smooth particle Mesh Ewald method. J. Chem. Phys. 103, 8577-8593 (1995).

63. Lee, J. et al. CHARMM-GUI input generator for NAMD, GROMACS, AMBER, OpenMM, and CHARMM/OpenMM simulations using the CHARMM36 additive force field. J. Chem. Theory Comput. 12, 405-413 (2016).

64. Van Der Spoel, D. et al. GROMACS: fast, flexible, and free. J. Comput. Chem. 26, 1701-1718 (2005).

65. Hess, B., Bekker, H., Berendsen, H. J. C. \& Fraaije, J. G. E. M. LINCS: a linear constraint solver for molecular simulations. J. Comput. Chem. 18, 1463-1472 (1997).

66. Jo, S., Lim, J. B., Klauda, J. B. \& Im, W. CHARMM-GUI membrane builder for mixed bilayers and its application to yeast membranes. Biophys. J. 97, 50-58 (2009).

67. Jo, S., Kim, T. \& Im, W. Automated builder and database of protein/ membrane complexes for molecular dynamics simulations. PLoS ONE 2, e880 (2007).

68. Hoover, W. G. Canonical dynamics: equilibrium phase-space distributions. Phys. Rev. A 31, 1695-1697 (1985).

69. Parrinello, M. \& Rahman, A. Polymorphic transitions in single-crystals-a new molecular-dynamics Method. J. Appl. Phys. 52, 7182-7190 (1981).

70. Hopkins, C. W., Le Grand, S., Walker, R. C. \& Roitberg, A. E. Long-time-step molecular dynamics through hydrogen mass repartitioning. J. Chem. Theory Comput. 11, 1864-1874 (2015).

\section{Acknowledgements}

This work was supported by the Creative-Pioneering Researchers Program through Seoul National University (H.-J.C.), the National Research Foundation of Korea funded by the Korean government (NRF-2020R1A2C2003783 and NRF-2019M3E5D6063903 to H.J.C.), and National Science Foundation, USA (MCB-2111728 to W.I.). This work was also supported by Korea Basic Science Institute grant (2020R1A6C101A183 to H.-J.C.) and the Korea Basic Science Institute under the R\&D program (Project No. C140440 to H.-J.C. and H.J.) supervised by the Ministry of Science and ICT. We thank Dr. Brian Kobilka (Stanford University) for providing the scFv16 construct, Chul Won Choi and Jungnam Bae (Seoul National University) for supporting grid preparation, Junsun Park and Dr. Soung-Hun Roh (Seoul National University) for providing materials and methods for grid screening. We also thank Dr. Bum Han Ryu (Institute for Basic Science (IBS), Korea) and Dr. Jin Seok Choi (KAIST Analysis Center for Research Advancement (KARA), Korea) for supporting grid screening at IBS and KARA cryo-EM facilities, respectively, and Global Science experimental Data hub Center (GSDC) at Korea Insti- tute of Science and Technology Information (KISTI) and the data analysis hub, Olaf at the IBS Research Solution Center for computing resources.

\section{Author contributions}

C.P. and J.K. contributed equally to this work. C.P. purified the NPY $-Y_{1} R-G_{i 1}-s c F v 16$ complex. J.K. collected and processed cryo-EM data with help from H.J. and I.B. C.P. and J.K. determined and refined the complex structure. S.B.K. performed cell-based assays including surface ELISA, BRET assays, and $\mathrm{Ca}^{2+}$ assays with help from J.K., S.A.K., and T.-Y.Y. Y.K.C. and W.I. performed MD simulations and H.W. and C.S. calculated the volume of ligand-binding pockets and generated a homology model of PP bound $\mathrm{Y}_{4}$ R. H.K. purified $\mathrm{G}_{\mathrm{il}}$ heterotrimer and scFv16. H.-J.C. conceived and directed the study. C.P., J.K., I.B., and H.-J.C. wrote the manuscript with contributions from all authors.

\section{Competing interests}

The authors declare no competing interests.

\section{Additional information}

Supplementary information The online version contains supplementary material available at https://doi.org/10.1038/s41467-022-28510-6.

Correspondence and requests for materials should be addressed to Hee-Jung Choi.

Peer review information Nature Communications thanks Cheng Zhang and the other, anonymous, reviewer(s) for their contribution to the peer review of this work. Peer reviewer reports are available.

Reprints and permission information is available at http://www.nature.com/reprints

Publisher's note Springer Nature remains neutral with regard to jurisdictional claims in published maps and institutional affiliations.

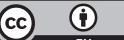

Open Access This article is licensed under a Creative Commons Attribution 4.0 International License, which permits use, sharing, adaptation, distribution and reproduction in any medium or format, as long as you give appropriate credit to the original author(s) and the source, provide a link to the Creative Commons license, and indicate if changes were made. The images or other third party material in this article are included in the article's Creative Commons license, unless indicated otherwise in a credit line to the material. If material is not included in the article's Creative Commons license and your intended use is not permitted by statutory regulation or exceeds the permitted use, you will need to obtain permission directly from the copyright holder. To view a copy of this license, visit http://creativecommons.org/ licenses/by/4.0/.

(C) The Author(s) 2022, corrected publication 2022 